\title{
Deletion of Autism Risk Gene Shank3 Disrupts Prefrontal Connectivity
}

\author{
Darco Pagani, ${ }^{1}$ Alice Bertero, ${ }^{1,2}$ Adam Liska, ${ }^{1}{ }^{\circledR}$ Alberto Galbusera, ${ }^{1}$ Mara Sabbioni, ${ }^{3}$ Noemi Barsotti, ${ }^{2}$ \\ Nigel Colenbier, ${ }^{4}$ Daniele Marinazzo, ${ }^{4}$ Maria Luisa Scattoni, ${ }^{3}$ Massimo Pasqualetti, ${ }^{1,2}$ and ${ }^{\circledR A l e s s a n d r o ~ G o z z i ~}{ }^{1}$ \\ ${ }^{1}$ Functional Neuroimaging Laboratory, Center for Neuroscience and Cognitive Systems, Istituto Italiano di Tecnologia, 38068 Rovereto, Italy, ${ }^{2}$ Department \\ of Biology, Unit of Cell and Developmental Biology, University of Pisa, 56126 Pisa, Italy, ${ }^{3}$ Department of Cell Biology and Neurosciences, Istituto Superiore \\ di Sanità, Neurotoxicology and Neuroendocrinology Section, 00161 Rome, Italy, and ${ }^{4}$ Department of Data Analysis, Ghent University, 9000 Ghent, Belgium
}

Mutations in the synaptic scaffolding protein SHANK3 are a major cause of autism and are associated with prominent intellectual and language deficits. However, the neural mechanisms whereby SHANK3 deficiency affects higher-order socio-communicative functions remain unclear. Using high-resolution functional and structural MRI in adult male mice, here we show that loss of Shank3 (Shank3B ${ }^{-1-}$ ) results in disrupted local and long-range prefrontal and frontostriatal functional connectivity. We document that prefrontal hypoconnectivity is associated with reduced short-range cortical projections density, and reduced gray matter volume. Finally, we show that prefrontal disconnectivity is predictive of social communication deficits, as assessed with ultrasound vocalization recordings. Collectively, our results reveal a critical role of SHANK3 in the development of prefrontal anatomy and function, and suggest that SHANK3 deficiency may predispose to intellectual disability and socio-communicative impairments via dysregulation of higher-order cortical connectivity.

Key words: autism spectrum disorder; basal ganglia; functional connectivity; mouse; Phelan-McDermid syndrome; resting state fMRI

Significance Statement

Mutations in the synaptic scaffolding protein SHANK3 are commonly associated with autism, intellectual, and language deficits. Previous research has linked SHANK3 deficiency to basal ganglia dysfunction, motor stereotypies, and social deficits. However, the neural mechanism whereby Shank3 gene mutations affects cortical functional connectivity and higher-order sociocommunicative functions remain unclear. Here we show that loss of SHANK3 in mice results in largely disrupted functional connectivity and abnormal gray matter anatomy in prefrontal areas. We also show that prefrontal connectivity disruption is tightly linked to socio-communicative deficits. Our findings suggest that SHANK3 is a critical orchestrator of frontocortical function, and that disrupted connectivity of prefrontal areas may underpin socio-communicative impairments observed in SHANK3 mutation carriers.

\section{Introduction}

Mutations in genes coding for synaptic proteins are among the most well-characterized genetic deficits observed in individuals with autism spectrum disorders (ASDs) (de la Torre-Ubieta et al.,

\footnotetext{
Received Oct. 2, 2018; revised Dec. 18, 2018; accepted April 16, 2019

Author contributions: M. Pagani, A.B., A.L., N.C., D.M., M.L.S., M. Pasqualetti, and A. Gozzi analyzed data; M. Pagani and A. Gozzi wrote the first draft of the paper; M. Pagani, M. Pasqualetti, and A. Gozzi edited the paper; M. Pagani and A. Gozzi wrote the paper; A.B., A. Galbusera, M.S., N.B., M.L.S., and M. Pasqualetti performed research; M. Pasqualetti and A. Gozzi designed research.

This work was supported by Simons Foundation Grants (SFARI 314688 and 400101) to A. Gozzi. A. Gozzi was also supported by Brain and Behavior Foundation 2017 (NARSAD - National Alliance for Research on Schizophrenia and Depression) and the European Research Council (ERC - DISCONN, GA802371). M. Pagani was supported by European Union's Horizon 2020 research and innovation programme (Marie Sklodowska-Curie Global Fellowship - CANSAS, GA845065).

The authors declare no competing financial interests.

Correspondence should be addressed to Alessandro Gozzi at alessandro.gozzi@iit.it.

https://doi.org/10.1523/JNEUROSCI.2529-18.2019

Copyright $\odot 2019$ the authors
}

2016). The postsynaptic scaffolding protein SH3 and multiple ankyrin repeat domains 3 (SHANK3) are a critical orchestrator of macromolecular signaling complex at glutamatergic synapses (Peca et al., 2011; Tabet et al., 2017). In humans, hemi-deletion of SHANK3 is found in a large fraction of Phelan-McDermid syndrome cases, a neurodevelopmental disorder characterized by ASD-like behaviors, intellectual disability (Phelan and McDermid, 2012), and profoundly impaired development of speech and language (Leblond et al., 2014; Monteiro and Feng, 2017). Recent genetic studies have also identified a large number of Shank3 mutations in ASD patients not diagnosed with PhelanMcDermid syndrome, strongly implicating disruption or mutations of SHANK3 as one of the most frequent and penetrant monogenic cause of ASD and socio-communicative impairments (Leblond et al., 2014).

Animal studies have provided insight into the circuital dysfunctions produced by Shank3 mutations. Prompted by the ini- 
tial observation of self-injurious grooming in mice lacking the SHANK3B isoform (Shank3B ${ }^{-1-}$ ) (Peca et al., 2011), multiple investigations have probed basal ganglia and corticostriatal function in mice harboring various Shank3 mutations. These efforts have highlighted impaired synaptic structure and transmission in striatopallidal medium spiny neurons of SHANK3-deficient mice (Peca et al., 2011), an effect that is partially rescued by functional stimulation (Wang et al., 2016) or adult tamoxifenmediated reexpression of $\alpha$ and $\beta$ isoforms in the affected neural populations (Mei et al., 2016). Functional deficits in corticostriatal synchronization and excitability have also been described in Shank3 mutant mice (Peixoto et al., 2016; Wang et al., 2016). For example, perseverative exploratory behaviors were described in mice with deletion of Shank3 in striatal inhibitory neurons, whereas self-grooming-induced lesions were observed in mice harboring Shank3 deletion in excitatory forebrain neurons (Bey et al., 2018). Further, a role of Shank3 has been identified in mediating mGlu5 receptor signaling in both the striatum and the cortex (Vicidomini et al., 2017). However, while many of these abnormalities have been convincingly linked to the motor stereotypies associated with Shank 3 disruption, the neural mechanisms whereby SHANK3 deficiency affects higher-order socio-communicative and cognitive functions remain unclear.

Systemic alterations in functional connectivity are prevalent in ASD (Di Martino et al., 2008) and have been regarded to account for the complex repertoire of symptoms exhibited by ASD patients (Vasa et al., 2016). In keeping with this notion, alterations in large-scale brain connectivity have been described in syndromic forms of autism, including Cntnap2 polymorphism (Scott-Van Zeeland et al., 2010) and chromosome 16p11.2 microdeletion (Bertero et al., 2018). Based on these observations, here we hypothesize that SHANK3 insufficiency may impact higher-order socio-communicative functions via a dysregulation of interregional functional connectivity. To probe this hypothesis, we used resting-state fMRI (rsfMRI) (Gozzi and Schwarz, 2016) and retrograde viral tracing (Bertero et al., 2018) to map functional and structural connectivity in Shank $3 B^{-1-}$ mice. This mouse line lacks the exons encoding the PDZ domain of SHANK3 leading to complete elimination of both $\mathrm{SHANK}_{\alpha}$ and SHANK $_{\beta}$ isoforms (Monteiro and Feng, 2017), and exhibits ASD-related behavioral abnormalities, including robust repetitive behavior and social interaction deficits (Peca et al., 2011). We show that homozygous loss of Shank $3 B$ results in disrupted frontocortical and frontostriatal connectivity, an effect associated with frontal gray matter (GM) hypotrophy and defective shortrange corticocortical wiring. We also document that prefrontal connectivity deficits are tightly linked to socio-communicative impairments, as assessed with ultrasound vocalizations (USVs) in a social interaction test. We suggest that Shank $3 B$ deletion may predispose to intellectual disability and socio-communicative impairments via a focal dysregulation of prefrontal connectivity.

\section{Materials and Methods}

\section{Ethical statement}

Animal studies were conducted in accordance with the Italian Law (DL 26/2014, EU 63/2010, Ministero della Sanità, Roma) and the recommendations in the National Institutes of Health Guide for the care and use of laboratory animals. Animal research protocols were reviewed and consented to by the animal care committee of the Istituto Italiano di Tecnologia and the Italian Ministry of Health specifically approved the study protocol (authorization 560/2016 PR to A. Gozzi). All surgical procedures were performed under anesthesia. $r s f M R I$

Male Shank3B ${ }^{-1-}(n=10,19-21$ weeks old, MGI \#1930016) and agematched Shank $3 B^{+/+}$control littermates $(n=11)$ were housed under controlled temperature $\left(21 \pm 1^{\circ} \mathrm{C}\right)$ and humidity $(60 \pm 10 \%)$. rsfMRI data were recorded as previously described (Ferrari et al., 2012; Sforazzini et al., 2016). Briefly, animals were anesthetized with isoflurane (5\% induction), intubated, and artificially ventilated ( $2 \%$ maintenance). The left femoral artery was cannulated for continuous blood pressure monitoring throughout imaging sessions and for arterial blood sampling. After surgery, isoflurane was discontinued and replaced with halothane $(0.7 \%)$. Functional data acquisition commenced $45 \mathrm{~min}$ after isoflurane cessation. Arterial blood gases $\left(\mathrm{p}_{\mathrm{a}} \mathrm{CO}_{2}\right.$ and $\left.\mathrm{p}_{\mathrm{a}} \mathrm{O}_{2}\right)$ were monitored at the end of the acquisition to exclude nonphysiological conditions. No intergroup differences were observed in mean $\mathrm{p}_{\mathrm{a}} \mathrm{CO}_{2}\left(\right.$ Shank $3 B^{+/+}: 16.5 \pm$ $4.0 \mathrm{mmHg}$; Shank3B $\left.{ }^{-/-}: 18.6 \pm 4.9 \mathrm{mmHg} ; p=0.29\right)$ or $\mathrm{p}_{\mathrm{a}} \mathrm{O}_{2}$ $\left(\right.$ Shank $3 B^{+/+}: 218.3 \pm 40.5 \mathrm{mmHg}$; Shank $3 B^{-l^{-}}: 219.5 \pm 29.7 \mathrm{mmHg}$; $p=0.94)$ between mutants and control mice. Possible genotypedependent differences in anesthesia sensitivity were evaluated with mean arterial blood pressure, amplitude of cortical BOLD signal fluctuations, and minimal alveolar concentration, three independent readouts previously shown to be correlated with anesthesia depth (Steffey et al., 2003; Liu et al., 2011; Zhan et al., 2014). To rule out a possible confounding neurovascular origin of the genotype-dependent rsfMRI connectivity alterations, we also calculated and compared the characteristic hemodynamic response function (Wu et al., 2013) in the PFC of both genotypes. Functional images were acquired with a 7T MRI scanner (Bruker, Biospin) as previously described (Liska et al., 2015), using a $72 \mathrm{~mm}$ birdcage transmit coil and a 4-channel solenoid coil for signal reception. For each session, in vivo anatomical images were acquired with a fast spin echo sequence $(\mathrm{TR}=5500 \mathrm{~ms}, \mathrm{TE}=60 \mathrm{~ms}$, matrix $192 \times 192$, FOV $2 \times 2 \mathrm{~cm}$, 24 coronal slices, slice thickness $500 \mu \mathrm{m}$ ). Cocentered single-shot BOLD rsfMRI time series were acquired using an EPI sequence with the following parameters: TR/TE $1200 / 15 \mathrm{~ms}$, flip angle $30^{\circ}$, matrix $100 \times 100$, FOV $2 \times 2 \mathrm{~cm}, 24$ coronal slices, slice thickness $500 \mu \mathrm{m}$ for 500 volumes.

\section{Functional connectivity analyses}

Functional connectivity based on rsfMRI is a method to map temporal dependency of spontaneous fluctuations of the BOLD signal between brain regions during no-task condition and is widely used in human clinical (Van Den Heuvel and Hulshoff Pol, 2010) and preclinical (Liska and Gozzi, 2016) studies to describe the macroscale functional organization of brain networks in autism and other neuropsychiatric disorders. Here we used rsfMRI connectivity to detect putative derangements of functional networks associated to Shank3B homozygous mutation.

Before calculating rsfMRI metrics, raw data were preprocessed as previously described (Sforazzini et al., 2016; Michetti et al., 2017; Liska et al., 2018). The initial 50 volumes of the time series were removed to allow for T1 equilibration effects. Data were then despiked, motion corrected, and spatially registered to a common reference template. Motion traces of head realignment parameters ( 3 translations +3 rotations) and mean ventricular signal (corresponding to the averaged BOLD signal within a reference ventricular mask) were used as nuisance covariates and regressed out from each time course. All rsfMRI time series also underwent bandpass filtering to a frequency window of $0.01-0.1 \mathrm{~Hz}$ and spatial smoothing with a FWHM of $0.6 \mathrm{~mm}$.

To obtain a data-driven identification of the brain regions exhibiting genotype-dependent alterations in functional connectivity, we calculated voxelwise long-range and local connectivity maps for all mice. Longrange connectivity is a graph-based metric also known as unthresholded weighted degree centrality and defines connectivity as the mean temporal correlation between a given voxel and all other voxels within the brain (Cole et al., 2010). Local connectivity was instead mapped by limiting the measurement of functional coupling within a 6-voxel radius sphere. To corroborate results of voxelwise connectivity, we calculated rsfMRI connectivity (Pearson correlation) between 170 unilateral cortical and subcortical anatomical regions (Janke et al., 2012) for each mouse.

Target regions of long-range connectivity alterations in Shank $3 B^{-1-}$ mice were mapped using seed-based analysis in ROIs or volumes of interest (VOIs). The location of small ROIs of $3 \times 3 \times 1$ voxels (Fig. 
$2 B-D$, small red squares) was selected based on between-group regional rsfMRI desynchronization observed with unbiased long-range connectivity. Specifically, seeds were placed in the anterior cingulate to map derangements in anteroposterior connectivity within the default mode network (DMN), and bilaterally in the striatum to probe impaired corticostriatal connectivity. Anteroposterior DMN and corticostriatal hypoconnectivity were probed by computing VOI-to-seeds correlations. PFC and striatum were used as VOIs for separate analysis. The location of seeds used for mapping is indicated in Figure $2 B-D$. Interhemispheric functional connectivity was delineated by computing correlation coefficients of ROI pairs covering major representative anatomical regions. Based on evidence that interhemispheric functional connectivity may be more heterogeneous in ASD patients with respect to age-matched typically developing populations (Hahamy et al., 2015), we also tested the presence of "idiosyncratic" interhemispheric connectivity in Shank $3 B^{-1-}$ mutants with respect to control mice, by measuring crosssubject variability in interhemispheric measurements of homotopic synchronization as described by Hahamy et al. (2015).

\section{Behavioral tests}

Behavioral testing was performed 2 weeks after the imaging sessions. Mice underwent a male-female social interaction test during the light phase, as previously described (Scattoni et al., 2011, 2013). An unfamiliar stimulus (i.e., a control female mouse in estrous) was placed into the home-cage of an isolated test male mouse, and social behavior was recorded during a 3 min test session. To measure USV recordings, an ultrasonic microphone (UltraSoundGate condenser microphone capsule CM16, Avisoft Bioacoustics) was mounted $20 \mathrm{~cm}$ above the cage, and the USVs were recorded using RECORDER software version 3.2 (Avisoft Bioacoustics; RRID:SCR_014436). Settings included sampling rate at $250 \mathrm{kHz}$; format 16 bit. The ultrasonic microphone was sensitive to frequencies between 10 and $180 \mathrm{kHz}$. For acoustical analysis, recordings were transferred to SASLabPro (version 4.40, Avisoft Bioacoustics; RRID:SCR_014438), and a fast Fourier transformation was conducted as previously described (Scattoni et al., 2008). Start times for the video and audio files were synchronized. Scoring of social investigation and other nonsocial behaviors was conducted using Observer 10XT software (Noldus Information Technology; RRID:SCR_004074), and multiple behavioral responses exhibited by the test mouse were measured: anogenital sniffing (direct contact with the anogenital area), total sniffing (sniffing or snout contact with the flank and head areas), following behaviors, self-grooming (self-cleaning, licking any part of its own body), mounting, rearing up against the wall of the home-cage, rearing, digging in the bedding, and immobility. Social investigation was defined as the sum of total sniffing and following behaviors (Scattoni et al., 2008). Sniffing and social investigations were quantified by computing their cumulative duration in seconds. USVs were quantified as number of recorded events during the entire behavioral session, and expressed as frequency.

\section{Structural MRI}

To identify putative GM reorganization coincident with functional hypoconnectivity in the cortex of Shank $3 B^{-1-}$ mice, we performed postmortem voxel-based morphometry (VBM) (Pagani et al., 2016b). Images were acquired ex vivo in PFA-fixed specimens, a procedure used to obtain high-resolution images with negligible confounding contributions from physiological or motion artifacts. Brains were imaged inside intact skulls to avoid postextraction deformations. Shank $3 B^{-1-}$ and control mice were deeply anesthetized with $5 \%$ isoflurane, and their brains were perfused in situ via cardiac perfusion (Dodero et al., 2013; Sannino et al., 2013). The perfusion was performed with PBS followed by $4 \%$ PFA ( 100 $\mathrm{ml}$, Sigma-Aldrich). Both perfusion solutions were added with a gadolinium chelate (ProHance, Bracco) at a concentration of 10 and $5 \mathrm{~mm}$, respectively, to shorten longitudinal relaxation times. High-resolution morpho-anatomical T2-weighted MR imaging of mouse brains was performed using a $72 \mathrm{~mm}$ birdcage transmit coil, a custom-built saddleshaped solenoid coil for signal reception. For each session, highresolution morpho-anatomical images were acquired with the following imaging parameters: FLASH 3D sequence with TR $=17 \mathrm{~ms}$, TE $=10 \mathrm{~ms}$, $\alpha=30^{\circ}$, matrix size of $260 \times 180 \times 180$, FOV of $1.83 \times 1.26 \times 1.26 \mathrm{~cm}$, and voxel size of $70 \mu \mathrm{m}$ (isotropic).

\section{VBM of $G M$}

Intergroup morpho-anatomical differences in local GM volumes were mapped using a registration-based VBM procedure (Pagani et al., 2016a, b; Pucilowska et al., 2018). Briefly, high-resolution T2-weighted images were corrected for intensity nonuniformity, skull stripped, and spatially normalized to a study-based template using affine and diffeomorphic registrations. Registered images were segmented to calculate tissue probability maps. The separation of the different tissues is improved by initializing the process with the probability maps of the study-based template previously segmented. The Jacobian determinants of the deformation field were extracted and applied to modulate the GM probability maps calculated during the segmentation. This procedure allowed the analysis of GM probability maps in terms of local volumetric variation instead of tissue density. Brains were also normalized by the total intracranial volume to further eliminate overall brain volume variations and smoothed using a Gaussian kernel with a $\sigma$ of 3 voxel width. To quantify volumetric changes identified with VBM, we used preprocessed images to independently calculate the size of neuroanatomical areas via volumetric anatomical labeling (Pagani et al., 2016b). To further corroborate the results of automatic volumetric mapping, we manually measured cortical thickness of the PFC in a coronal slice (corresponding approximately to $Z$ bregma $-1.1 \mathrm{~mm}$ ) by using the ruler tool available in the ITK Workbench (RRID:SCR_002010).

\section{Diffusion MRI}

Fixed brains also underwent diffusion-weighted (DW) imaging. Each DW dataset was composed of 8 non-DW images and 81 different diffusion gradient-encoding directions with $b=3000 \mathrm{~s} / \mathrm{mm}^{2}(\partial=6 \mathrm{~ms}, \Delta=$ $13 \mathrm{~ms}$ ) acquired using an EPI sequence with the following parameters: $\mathrm{TR} / \mathrm{TE}=13,500 / 27.6 \mathrm{~ms}$, FOV $1.68 \times 1.54 \mathrm{~cm}$, matrix $120 \times 110$, in-plane spatial resolution $140 \times 140 \mu \mathrm{m}, 54$ coronal slices, slice thickness $280 \mu \mathrm{m}$, number of averages 20 as recently described (Dodero et al., 2013; Liska et al., 2018).

White matter fiber tractography and tract-based spatial statistics The DW datasets were first corrected for eddy current distortions and skull-stripped. The resulting individual brain masks were manually corrected using ITK-SNAP (Yushkevich et al., 2006). Whole-brain tractography was performed with MRtrix3 (Tournier et al., 2012) (RRID: SCR_006971) using constrained spherical deconvolution $(\operatorname{lmax}=8)$ and probabilistic tracking (iFOD2) with an FOD amplitude cutoff of 0.2. For each dataset, the whole-brain mask was used as a seed, and a total of 100,000 streamlines were generated. The corpus callosum and cingulum were selected as tracts of interest, given their major corticocortical extension and direct involvement in prefrontal-posterior connectivity. The tracts were virtually dissected with waypoint VOIs previously described (Liska et al., 2018) using TrackVis (RRID:SCR_004817). For visualization purposes, dissected corpus callosum and cingulum were transformed to the Allen Mouse Common Coordinate Framework, version 3 (http://www.brain-map.org/). Color encoding indicates the preferred fiber direction, with red denoting left-right, blue denoting back-front, and green denoting up-down (i.e., through the image plane). Further, we performed a Tract-Based Spatial Statistics analysis, as implemented in FSL (Smith et al., 2006) and previously described (Dodero et al., 2013). Fractional anisotropy (FA) maps from all subjects were nonlinearly registered to an in-house FA template with FSL-FLIRT and FSL-FNIRT and thinned using an FA threshold of 0.2 to create a skeleton of the white matter.

\section{Virus production and injection}

Unpseudotyped recombinant SAD $\Delta$ G-mCherry rabies virus was produced as previously described (Bertero et al., 2018). Briefly, B7GG packaging cells, which express the rabies envelope G-protein, were infected with unpseudotyped SAD $\Delta \mathrm{G}-\mathrm{mCherry}-\mathrm{RV}$, obtained by courtesy of Prof. Edward Callaway. Five to $6 \mathrm{~d}$ after infection, viral particles were collected, filtrated through $0.45 \mu \mathrm{m}$ filter, and concentrated by two rounds of ultracentrifugation. The titer of the SAD $\Delta$ G-mCherry-RV preparation was established by infecting Hek-293T cells (ATCC, catalog \#CRL-11268) with 10-fold serial dilution of viral stock, counting mCherry-expressing cells $3 \mathrm{~d}$ after infection. The titer was calculated as 
$2 \times 10^{11}$ infective units $/ \mathrm{ml}(\mathrm{IU} / \mathrm{ml})$, and the stock was therefore considered suitable for in vivo microinjection. Mice were anesthetized with isoflurane (4\%) and firmly stabilized on a stereotaxic apparatus (Stoelting). A micro drill (Cellpoint Scientific) was used to drill holes through the skull. Retrograde viral (RV) injections were performed as previously described in adult (12- to 16-week-old) male Shank $3 B^{-1-}$ and control Shank $3 B^{+/+}$littermates (Cavaccini et al., 2018). Injections were performed with a Nanofil syringe mounted on an UltraMicroPump UMP3 with a four channel Micro4 controller (World Precision Instruments; RRID:SCR_008593), at a speed of $5 \mathrm{nl}$ per second, followed by a 5-10 min waiting period, to avoid backflow of viral solution and unspecific labeling; $1 \mu \mathrm{l}$ of viral stock solution was injected unilaterally in the primary cingulate cortex of 10- to 20-week-old Shank $3 B^{-1-}$ and WT control mice. Coordinates for injections from bregma are as follows: $1.42 \mathrm{~mm}$ from anterior to posterior, $0.3 \mathrm{~mm}$ lateral, and $-1.6 \mathrm{~mm}$ deep.

\section{Immunohistochemistry and image analysis}

Seven days after RV injection, animals were transcardially perfused with $4 \%$ PFA under deep isoflurane anesthesia (5\%), brains were dissected, postfixed overnight at $4^{\circ} \mathrm{C}$, and vibratome-cut (Leica Microsystems; RRID:SCR_008960). RV-infected cells were detected by means of immunohistochemistry performed on every other $100-\mu$ m-thick coronal section, using rabbit anti-red fluorescent protein primary antibody (1:500, Abcam; RRID:AB_945213) and goat anti rabbit-HRP secondary antibody (1:500, Jackson ImmunoResearch Laboratories), followed by DAB (Sigma-Aldrich) staining. Wide-field imaging was performed with a MacroFluo microscope (Leica Microsystems), and RGB pictures were acquired at $1024 \times 1024$ pixel resolution. Labeled neuron identification was manually performed by an operator blind to the genotype, while the analysis was performed using custom-made scripts to automatically register each brain section on the corresponding table on the Allen Brain Atlas (Dong, 2008) and to count labeled neurons and assign them to their anatomical localization. Final regional cell population counts were expressed as fraction of the total amount of labeled cells counted across the entire brain (including both hemispheres) for each animal. To probe the excitatory or inhibitory nature of rabies-positive cells, $100 \mu \mathrm{m}$ brain coronal sections from the same animals were incubated in blocking solution $5 \%$ horse serum $/ 0.5 \%$ PB Triton-X for $1 \mathrm{~h}$ at room temperature. Slices were next incubated overnight with a rabbit anti-GABA primary antibody (1:1000, Sigma-Aldrich; RRID:AB_477652) at $4^{\circ} \mathrm{C}$. AlexaFluor488-conjugated goat anti-rabbit (1:500, Thermo Fisher Scientific; RRID: AB_2576217) was used as secondary antibody after a further overnight incubation at $4^{\circ} \mathrm{C}$. The following day, sections were washed three times with $0.5 \%$ PB Triton-X and mounted with Aqua-Poly/mount mounting medium (Polysciences). High-power confocal images were obtained on a Nikon A1 confocal microscope with a $20 \times$ plan-apochromat objective.

\section{$\mathrm{NeuN}^{+}$cell density quantification}

Six anatomically comparable $100-\mu$ m-thick coronal sections of Shank $3 B^{-1-}$ and control littermates were processed for NeuN immunostaining and neural density quantification in the ACC. Immunofluorescence on free floating sections was performed using mouse anti NeuN primary antibody (1:1000, Millipore; RRID:AB_2298772), overnight, followed by $2 \mathrm{~h}$ rhodamine-red goat anti-mouse secondary antibody (1:500, Invitrogen, R6393). Images were acquired on a Nikon A1 confocal system, equipped with 561 diode. $Z$ series of 5 stacks ( $1 \mu \mathrm{m} z$ step) confocal images, using a $10 \times$ plan-apochromat objective, were acquired at $1024 \times 1024$ pixel resolution. NeuN-positive cells in the ACC were manually counted by an operator blind to the genotype, using the Cell Counter plugin of Fiji (RRID:SCR_002285) (Schindelin et al., 2012). Cell count for each section was normalized on the number of pixels in the area. Data are expressed as mean number of $\mathrm{NeuN}^{+}$cells every 100 pixels per genotype.

\section{Experimental design and statistical analyses}

RsfMRI was performed in adult male Shank $3 B^{-1-}$ mice $(n=10)$ and age-matched Shank $3 B^{+/+}$control littermates $(n=11)$. The same animals also underwent behavioral testing and ex vivo structural and diffusion imaging. Viral retrograde labeling and GABA immunostaining were performed on a separate cohort of adult male Shank $3 B^{-1-}(n=4)$ and
Shank3B ${ }^{+/+}(n=5)$ mice. $\mathrm{NeuN}^{+}$cell density quantification was performed on adult male Shank $3 B^{-1-}$ mice and control mice $(n=5$, each group).

rsfMRI. In global and local rsfMRI connectivity mapping as well as all seed-based analyses, Pearson's correlation scores were first transformed to $z$ scores using Fisher's $r$-to- $z$ transform. Voxelwise intergroup differences were then assessed using an unpaired two-tailed Student's $t$ test $(t>2, p<0.05)$ followed by a family-wise error (FWER) cluster correction using a cluster threshold of $p=0.01$ (Worsley et al., 1992) as implemented in FSL (Jenkinson et al., 2012).

Brainwide rsfMRI mapping in anatomically parcellated regions was performed using an unpaired two-tailed Student's $t$ test for each element of the corresponding correlation matrix separately $(t>3.5, p<0.001)$. FWER correction at the network level was performed using 5000 random permutations $(p<0.05)$ as implemented in the network-based statistics (NBS) (Zalesky et al., 2010). The statistical significance of intergroup effects in VOI-to-seeds analysis was quantified using two-way repeatedmeasures ANOVA, where seed location (repeated-measure factor) and genotype (between-group factor) were used as input variables. Intergroup statistics of interhemispheric and idiosyncratic connectivity, as well as measures to exclude nonphysiological conditions due to anesthesia sensitivity, were performed with two-tailed Student's $t$ test $(t>2, p<$ 0.05).

Structural imaging. Voxelwise intergroup differences in regional GM volume were mapped using a two-tailed Student's $t$ test $(t>2, p<0.05)$ followed by an FWER cluster correction using a cluster threshold of $p=$ 0.01 as implemented in FSL. Between-genotype differences in automated volumetric labeling of GM volume and manual measurements of cortical thickness were statistically assessed using a two-tailed Student's $t$ test $(t>$ $2, p<0.05)$.

Diffusion imaging. Intergroup differences in streamline counts of white matter tracts were assessed using a two-tailed Student's $t$ test $(t>2$, $p<0.05$ ). Voxelwise intergroup differences in FA between mutant and control mice were evaluated via permutation testing using 5000 permutations $(p<0.05)$ (Winkler et al., 2014). FA was also compared between Shank $3 B^{-1-}$ mice and controls in representative regions, such as corpus callosum, dorsal hippocampal commissure, and forceps minor using a two-tailed Student's $t$ test $(t>2, p<0.05)$.

Behavioral tests and immunochemistry. Statistical analyses of behavioral and immunohistochemistry data were performed with Prism 8.0 (GraphPad; RRID:SCR_002798). Genotype-dependent differences in socio-communicative behaviors where assessed with unpaired two-tailed Student's $t$ test followed by a false discovery rate (FDR) correction for multiple comparisons $(q=0.05)$. Voxelwise correlation mapping between seed-based functional connectivity and behavioral scores was performed with Pearson's correlation with $p<0.01$, FWER clustercorrected, with cluster defining threshold of $t>2(p<0.05)$. Intergroup differences in retrogradely labeled cells in prefrontal areas and in distal source regions, as well as $\mathrm{NeuN}^{+}$and $\mathrm{RBV}^{+} / \mathrm{GABA}^{+}$cells, were assessed using a two-tailed Student's $t$ test $(t>2, p<0.05)$. To control for multiple distal source regions, we applied an FDR correction $(q=0$ $0.05)$.

\section{Results}

\section{Disrupted prefrontal connectivity in Shank3B-null mice}

To identify regions exhibiting altered rsfMRI connectivity in Shank $3 B^{-1-}$ mice, we computed intergroup differences in longrange and local functional connectivity using spatially unbiased aggregative network-based metrics. These analyses revealed a robust reduction in long-range and local connectivity in prefrontal cortical areas of Shank $3 B^{-/-}$mutants $(t$ test, $p<0.01$, FWER cluster-corrected, with cluster defining threshold of $t>2, p<$ 0.05 ; Fig. $1 A, B$, top). Long-range connectivity reductions appeared to be more widespread than corresponding local connectivity deficits and included retrosplenial and prefrontal-infralimbic cortices, as well as bilateral striatal areas and anterior insular cortices (Fig. $1 A$, top). Post hoc region-based quantifications confirmed these effects (Fig. $1 A, B$, bottom, $t$ test, $p<0.05$ ). To corroborate these 
A

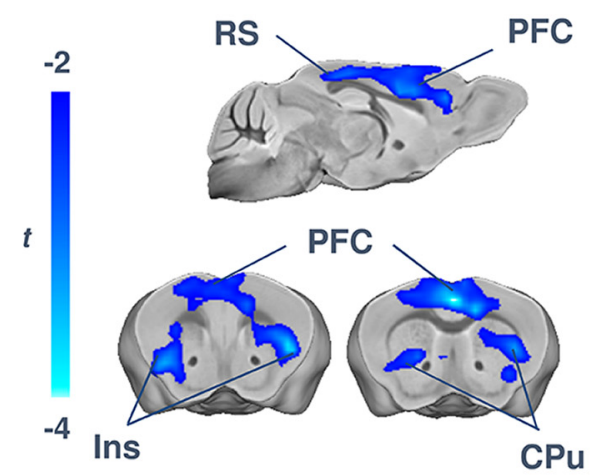

B

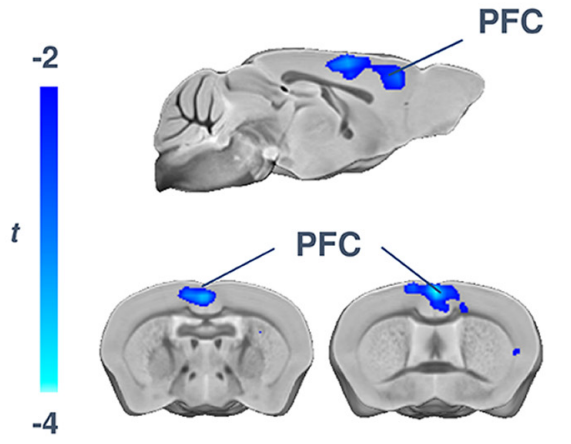

C
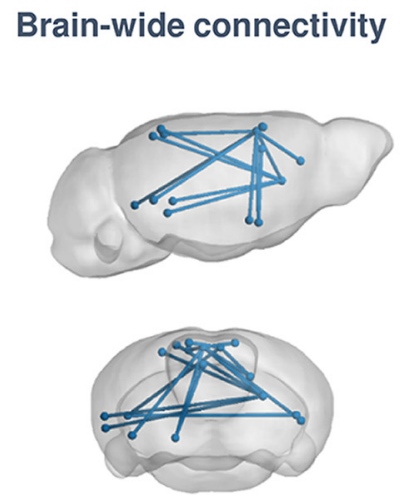
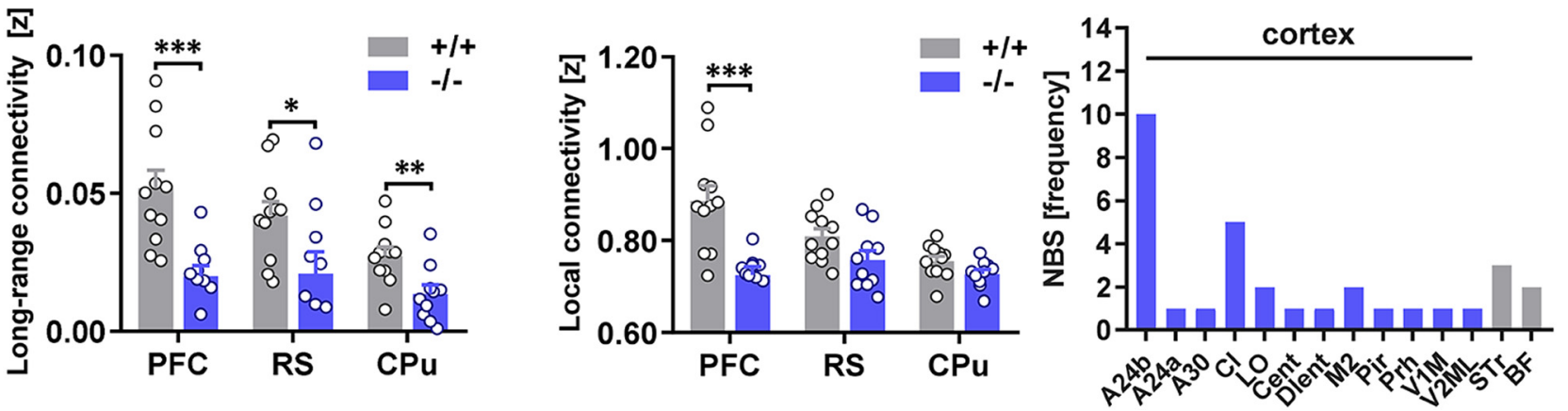

Figure 1. Reduced local and long-range connectivity in prefrontal areas of Shank3B ${ }^{-1-}$ mice. Areas exhibiting significantly reduced $(\boldsymbol{A})$ long-range and $(\boldsymbol{B})$ local functional connectivity in Shank3B ${ }^{-1-}$ mutants (blue, light/blue). PFC, prefrontal cortex; CPu, Caudate-putamen; RS, retrosplenial cortex. ${ }^{*} p<0.05,{ }^{* *} p<0.01,{ }^{* * *} p<0.001$. Error bars represent SEM. C, Intergroup comparison of rsfMRI connectivity in regionally parcellated brains using NBS. Plot represents the number of links exhibiting reduced rsfMRI connectivity identified with NBS. A24a, Prefrontal area 24a; A24b, prefrontal area 24b; A30, prefrontal area 30; Cl, claustrum; L0, lateral orbitofrontal; Cent, caudomedial entorhinal cortex; DLMent, dorsolateral-medial entorhinal cortex; M2, secondary motor cortex; Pir, piriform cortex; Prh, perirhinal cortex; V1M, medial primary visual cortex; V2ML, mediolateral secondary visual cortex; STr, subiculum transition area; BF, basal forebrain.

findings and probe long-range targets of the observed functional hypoconnectivity, we next calculated genotype differences in interregional rsfMRI connectivity by applying NBS to a wholebrain network parcellation (Zalesky et al., 2010). This analysis confirmed the presence of predominant regional hypoconnectivity in prefrontal regions and cingulate-retrosplenial midline areas ( $t$ test, linkwise threshold $t>3.5$, FWER-corrected at $p<0.05$; Fig. $1 C$ ). It also revealed the presence of long-range desynchronization between prefrontal regions and other long-range cortical targets, including entorhinal, piriform, and motor cortices (Fig. 1C). These results reveal the presence of impaired functional synchronization in prefrontal cortical areas of Shank3B-1mice.

\section{Reduced default-mode-network and frontostriatal connectivity in Shank3B ${ }^{-/-}$mice}

Long-range connectivity mapping revealed foci of weaker synchronization in PFC and retrosplenial cortex of Shank $3 B^{-1-}$ mice, an ensemble of associative regions that act as pivotal nodes of the mouse DMN (Sforazzini et al., 2014). To probe the functional integrity of this network, we performed a seed-based correlation analysis along its midline extension. In keeping with global connectivity mapping, we observed foci of reduced connectivity throughout the anteroposterior cingulate-retrosplenial axis of the DMN ( $t$ test, $p<0.01$, FWER cluster-corrected, with cluster defining threshold of $t>2, p<0.05$ ), as well as reduced involvement of temporal cortical areas in Shank $3 B^{-1-}$ mice (Fig. $2 A)$. A quantification of DMN connectivity via multiple prefrontal-DMN seed pairs (Fig. 2B) revealed a clear disconnec- tion of the PFC with the rest of this network (two-way repeatedmeasures ANOVA, genotype effect, $F_{(1,19)}=15.01, p=0.001$; Fig. $2 B$ ). Similarly, seed-based mapping of the anterior caudateputamen, a region exhibiting focal underconnectivity (Fig. 1A), revealed genotype-dependent functional desynchronization between basal ganglia and the ACC $\left(t\right.$ test, $t_{(19)}=2.30, p=0.03$; Fig. $2 C, D)$. Striatal regions also exhibited robustly decreased interhemispheric connectivity $\left(t\right.$ test, $t_{(19)}=2.90, p=0.009$; Fig. $3 A, B)$. Interhemispheric connectivity between motor-sensory cortical networks was otherwise largely unimpaired, supporting the network specificity of these findings (Fig. $3 A, B$ ). Interestingly, we found Shank3B $B^{-1-}$ mutants to exhibit decreased intersubject similarity as assessed with group-based homotopic patterns (Fig. $4 A, B$ ), a finding recapitulating idiosyncratic interhemispheric connectivity patterns in human ASD patients ( $\mathrm{Ha}-$ hamy et al., 2015). This effect was especially prominent in cortical regions (Fig. $4 C, D$ ).

Importantly, no genotype-dependent differences in anesthesia sensitivity were detected when assessed with mean arterial blood pressure mapping ( $t$ test, $t_{(19)}=1.23 p=0.23$; Fig. $5 A$ ), amplitude of cortical BOLD signal fluctuations ( $t$ test, $t_{(19)}=$ $1.15, p=0.26$; Fig. $5 B)$, and minimal alveolar concentration of anesthetic ( $t$ test, $t_{(19)}=1.02, p=0.31$; Fig. $5 C$ ), three physiological measures sensitive to anesthesia depth (Steffey et al., 2003; Liu et al., 2011; Bertero et al., 2018). We similarly did not observe any intergroup differences in the mean height $\left(t\right.$ test, $t_{(17)}=0.11$; $p=0.91)$, FWHM ( $t$ test, $\left.t_{(17)}=1.19 ; p=0.25\right)$, or time to peak ( $t$ test, $\left.t_{(17)}=0.11 ; p=0.91\right)$ of the hemodynamic response, further corroborating a neural (rather than neurovascular) origin 


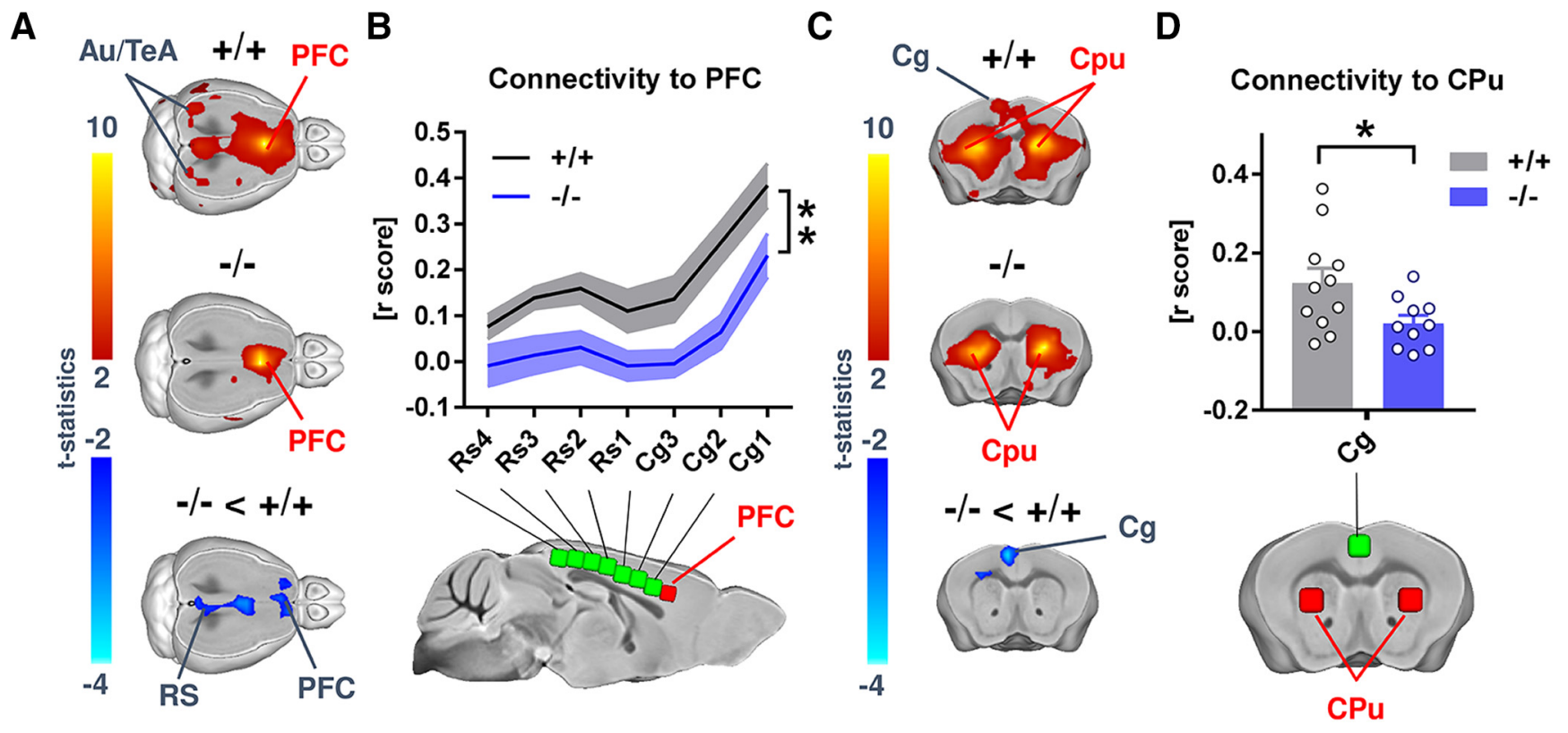

Figure 2. Prefrontal and frontostriatal underconnectivity in Shank3B-1- mice. $A$, Spatial extension of the regions exhibiting significant long-range connectivity with the PFC in Shank3B control $(+/+$, top) and mutant $(-/-)$ mice. Red-yellow represents area of significant correlation (i.e., functional connectivity) with PFC seed region (red lettering). Blue-light blue represents regions exhibiting significantly reduced connectivity with the PFC in Shank3B ${ }^{-1-}$ mice with respect to Shank3B ${ }^{+/+}$littermates $(-/-<+/+$, bottom). $B$, Anteroposterior profiling of rsfMRI connectivity of the PFC with respect to cingulate-retrosplenial component of mouse DMN. Note the reduced connectivity throughout the anteroposterior extension of the mouse DMN in Shank3B mutants. C, Seed-based connectivity maps of the striatum in Shank3b control mice and mutants. Red-yellow represents significant rsfMRI functional connectivity with striatal seed regions in control $(+/+$, top) and mutant Shank3B mice $(-/-$, middle). Blue-light blue represents foci of significantly reduced striatal connectivity in Shank3B $-/-$ mutants $(-/-<+/+$, bottom, seed, red lettering). $D$, Frontostriatal rsfMRI desynchronization in Shank3B ${ }^{-1-}$ mice. The effects were quantified in a reference volume of interest (green) placed in the ACC. Red represents striatal seed regions. Error bars represent SEM. Au, Auditory cortex; Cg, cingulate cortex; Cpu, striatum, RS, retrosplenial; TeA, temporal associative cortex. ${ }^{*} p<0.05,{ }^{* *} p<0.01$.

of the mapped changes (Fig. 5D). Together with the observation of region-dependent rsfMRI alterations, these findings argue against a significant confounding contribution of anesthesia to the observed hypoconnectivity phenotype.

\section{Prefrontal underconnectivity and socio-communicative deficits in Shank $3 B^{-1-}$ mice are tightly correlated} SHANK3 deficiency in humans is associated with marked language and communicative deficits. To test whether Shank3 mutations can similarly affect socio-communicative function in rodents and identify the neural circuits responsible for this phenomenon, we measured sociability and USVs in a male-female interaction test, and we correlated each of these measurements with rsfMRI connectivity metrics we previously recorded in the same subjects. Behavioral testing revealed unaltered total sniffing and social communication in Shank $3 B^{-1-}$ mice, along with a trend for reduced sociability as assessed with anogenital sniffing duration ( $t$ test, $t_{(19)}=2.21, p=0.04$ uncorrected; Fig. $6 A$ ). The same recordings revealed a marked reduction in the frequency of USVs in Shank3B $B^{-/-}$mice ( $t$ test, $t_{(19)}=3.60, p=0.002$, FDRcorrected; Fig. $6 B$ ). To probe whether prefrontal rsfMRI hypoconnectivity could be related to the socio-communicative impairments observed in the same mice in post-MRI behavioral tests, we calculated voxelwise correlation between vocalization scores and individual prefrontal rsfMRI connectivity maps. This analysis revealed that prefrontal hypoconnectivity of the DMN was prominently associated with reduced vocalizations, with evidence of robust foci of correlation in prefrontal and cingulate cortices ( $t$ test, $p<0.01$, FWER cluster-corrected, with cluster defining threshold of $t>2, p<0.05$; Fig. $6 C$ ). A regional quantification of this effect in the PFC confirmed a strong positive correlation between these two readouts $(r=0.67, p<0.001$; Fig.
$6 D)$, corresponding to an explained variance $\left(R^{2}\right)$ of 0.45 . These findings reveal a robust correlation between prefrontal hypoconnectivity and socio-communicative deficits in Shank $3 B^{-1-}$ mice. Voxelwise correlation mapping did not reveal instead any significant association between USV frequency and corticostriatal rsfMRI connectivity ( $t$ test, $p>0.01$, FWER cluster-corrected, with cluster defining threshold of $t>2 p<0.05$ ), corroborating the circuital specificity of these findings.

\section{Reduced prefrontal GM volume in Shank $3 B^{-/-}$mice}

To investigate whether Shank3B deficiency in mice produces brainwide cortical neuroanatomical alterations, we used highresolution structural MRI to obtain spatially unbiased maps of GM volume with voxel resolution (Pagani et al., 2016a). Notably, intergroup comparisons revealed extended reductions in cortical GM in Shank3B $B^{-1-}$ mice, encompassing a network of cortical regions exhibiting remarkable neuroanatomical overlap with prefrontal, midline, and temporal nodes of the mouse DMN $(t$ test, $p<0.01$, FWER cluster-corrected defining threshold of $t>$ $2, p<0.05$; Fig. $7 A$ ). In keeping with this finding, manual cortical thickness measurements on high-resolution anatomical scans revealed a statistically significant thinning of the PFC in Shank $3 B^{-1-}$ mice (Fig. $7 B$ ) when independently measured along two independent axes (vertical, $t$ test, $t_{(19)}=2.95, p=0.008$; oblique, $t$ test, $t_{(19)}=2.39, p=0.027$ ).

Atlas-based volumetric estimation of regional GM volume confirmed the presence of reduced GM volume in cortical constituents of the mouse DMN ( $t$ test, $p<0.05$; Fig. 7C). Interestingly, we found prefrontal GM volume to be significantly correlated with long-range connectivity and USV frequency (Fig. $7 D ; r=0.592, p=0.009$, and $r=0.521, p=0.022$, respectively), but not with short-range connectivity $(r=0.119, p=0.636)$, 


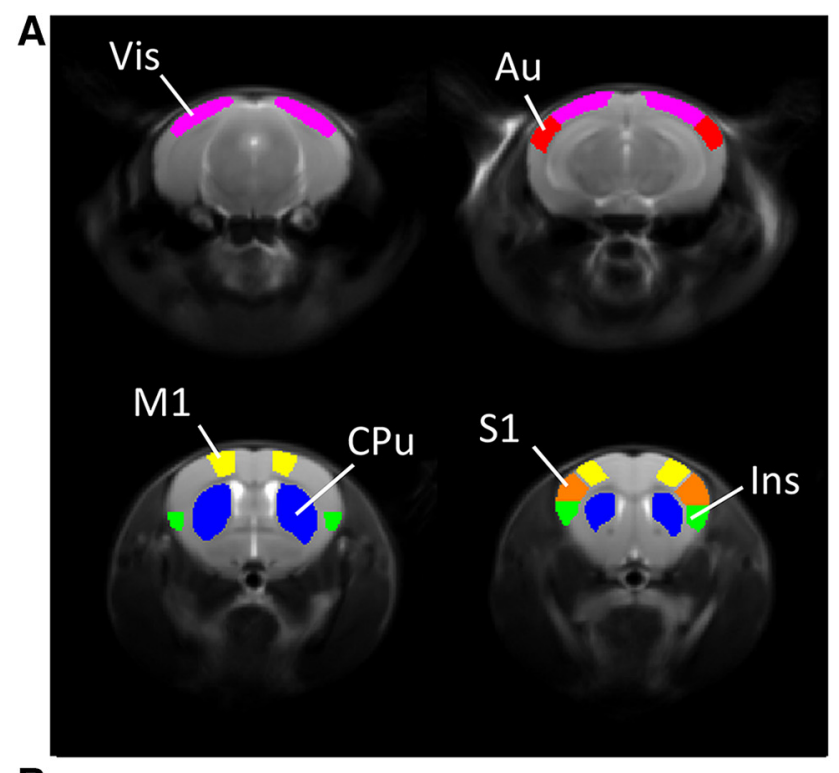

B

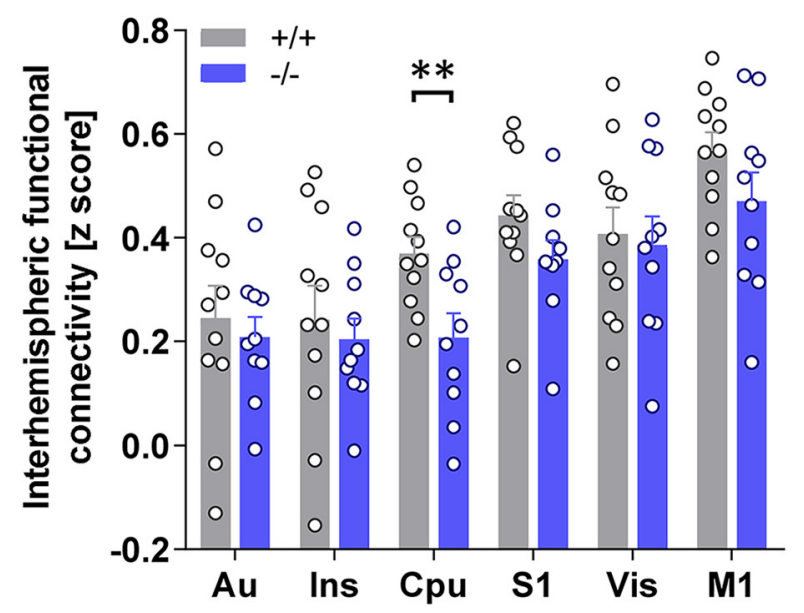

Figure 3. Reduced interhemispheric striatal connectivity in Shank3B-1- mice. Interhemispheric rsfMRI connectivity calculated between time courses extracted from representative VOls depicted in $\boldsymbol{A}$. The resulting interhemispheric $r$ scores were transformed to $z$ scores using Fisher's $r$-to-z transform (B). Au, Auditory cortex; Ins, insular cortex; $\mathrm{CPu}$, striatum; $\mathrm{S1}$, primary somatosensory cortex; Vis, visual cortex; M1, motor cortex. Error bars represent SEM. ${ }^{* *} p<0.01$.

pointing at a putative unifying mechanistic link between GM hypotrophy, disrupted rsfMRI connectivity, and impaired USVs in Shank3B $B^{-1-}$ mice.

Reduced short-range projection density in Shank $3 B^{-/-}$mice Recent studies have revealed mesoscale wiring alterations as a possible correlate for long-range functional desynchronization in genetic models of autism (Bertero et al., 2018; Liska et al., 2018). To investigate the presence of neuroanatomical alterations in prefrontal areas of Shank3B mutant mice, we first examined whether these alterations could be related to differences in neuronal density, similar to findings recently described in a primate model of Shank deficiency (Zhao et al., 2017). Postmortem quantification of neuronal somas in the medial PFC did not reveal genotype-dependent differences in NeuN-positive cell density $\left(\right.$ Shank $3 B^{+/+}: 0.43 \pm 0.06$ cells/100pixel; Shank3B ${ }^{-/-}: 0.48 \pm$ 0.06 cells/100pixel; $p=0.27$, two-tailed $t$ test).

We next performed monosynaptic retrograde tracing of the PFC using a recombinant retrograde rabies virus and quantified the fraction of retrogradely labeled cells within prefrontal areas exhibiting significant local rsfMRI hypoconnectivity (Fig. $8 A$ ), and in a representative set of distal source regions (Fig. 8B). Quantifications of labeled cells within the prefrontal foci of hypoconnectivity revealed a significantly reduced number of locally projecting neurons in Shank3B mutants ( $t$ test, $t_{(7)}=3.90, p=$ 0.025 ; Fig. $8 A$, right). No intergroup difference in the number of prefrontal-projecting neurons was observed in any of the longrange source regions ( $p>0.05$, all regions, FDR-corrected). Subsequent immunostaining showed that the vast majority of rabies-positive cells in both genotypes $(96.7 \pm 1.6 \%$ and $98.4 \pm$ $1.4 \%$, respectively, $p=0.34$; Fig. $8 C$ ) do not exhibit GABApositive staining. This finding suggests that the mesoscale wiring defects observed in the PFC of Shank3B mutants predominantly involve excitatory neuronal populations.

Finally, we probed large-scale white matter microstructural integrity using diffusion-weighted MRI and performed ex vivo tractography to virtually dissect the corpus callosum and cingulum, two major fiber bundles linking interhemispheric and anteroposterior cortical areas. No genotype-dependent white matter reorganization $(p>0.05$; Fig. $8 D)$ or microstructural alterations (fractional anisotropy, median diffusivity, radial diffusivity, $p>0.05$, all parameters voxelwise) were observed. These findings argue against a contribution of gross white matter reorganization to the observed functional disconnectivity.

\section{Discussion}

Here we document disrupted frontocortical functional connectivity in Shank3B-deficient mice, and show that this trait is tightly linked to impaired socio-communicative functions. We also describe reduced frontocortical volume and short-range neuronal wiring as plausible mesoscopic correlates of the observed disconnectivity. These findings establish a link between a prevalent monogenic form of ASD and aberrant neocortical connectivity, suggesting that Shank3 deficiency can predispose to sociocommunicative dysfunction and intellectual disability via dysregulation of higher-order cortical synchronization.

Disruption in large-scale brain network activity has been consistently observed in individuals with autism, and is regarded to partially account for language and cognitive impairments manifested in ASD (Vasa et al., 2016). However, the clinical heterogeneity of ASD has so far precluded a systematic and reliable identification of network alterations specific to distinctive pathophysiological or genetic etiologies. Our results suggest that Shank3 deficiency, one of the most common genetic alterations in ASD patients, leads to selective desynchronization of associative and integrative cortical regions.

This finding adds to recent human and animal evidence implicating disrupted functional connectivity as a network-level alteration of pathophysiological relevance for ASD, a feature that has been observed in an increasing number of syndromic forms of ASD, such as cortical dysplasia produced by CNTNAP2 (Liska et al., 2018), 16p11.2 chromosomal microdeletion (Bertero et al., 2018), haploinsufficiency in chromatin domain 8 protein (Chd8) (Suetterlin et al., 2018), and genetic variants in Met receptor tyrosine kinase (Rudie et al., 2012). Notably, cross-comparisons of these studies are revealing shared connectional signatures of etiological relevance for ASD. For example, converging patterns of prefrontal hypoconnectivity have been shown in mouse models and human carriers of chromosomal 16p11.2 microdeletion (Bertero et al., 2018) or mutations in the cell-adhesion protein CNTNAP2 (Scott-Van Zeeland et al., 2010; Liska et al., 2018). Disrupted functional connectivity in prefrontal cortical regions have also been reported in mouse lines recapitulating other 
A

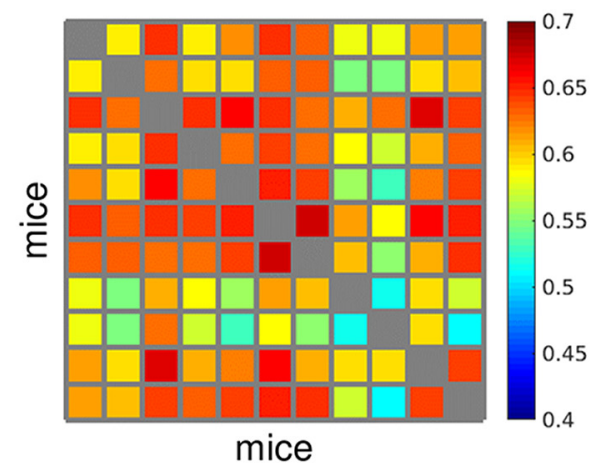

C

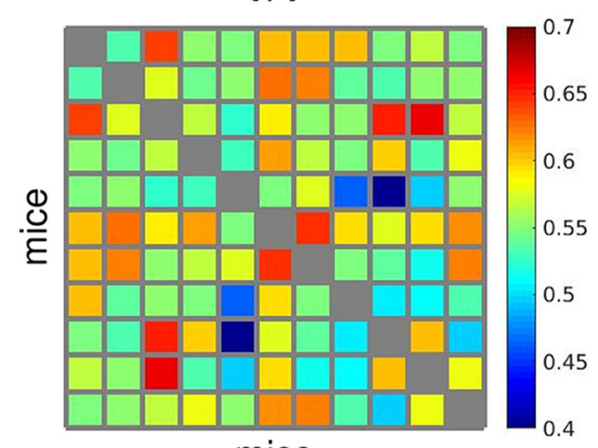

mice
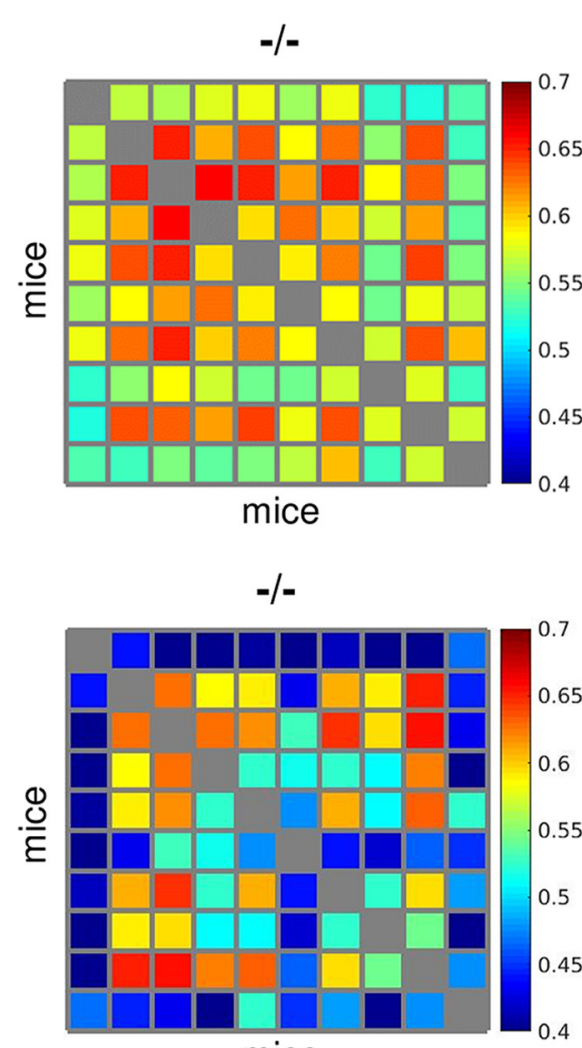

B

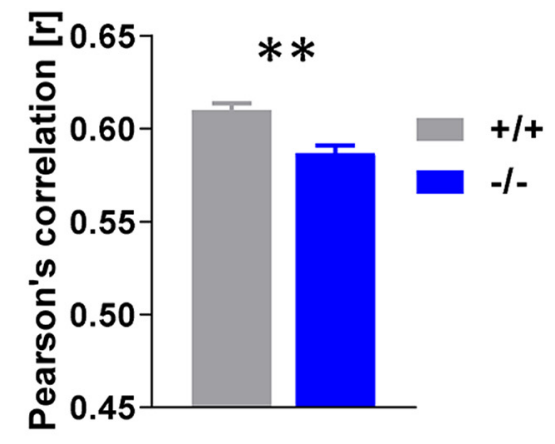

D

Cerebral Cortex

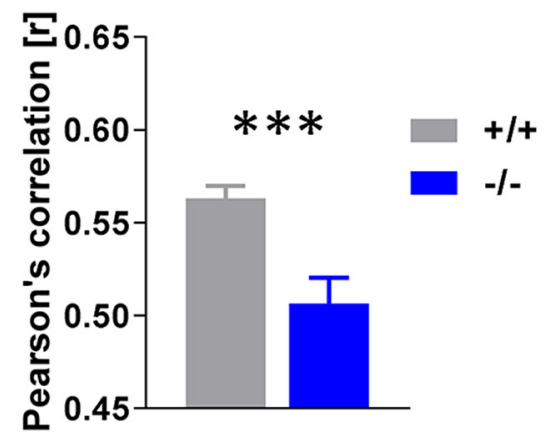

Figure 4. Idiosyncratic interhemispheric connectivity in Shank $3 B^{-1-}$ mice. Each element of the matrix represents the intersubject Pearson's correlation value between a pair of homotopic interhemispheric connectivity maps obtained from each individual subject. Voxelwise homotopic connectivity maps were calculated for each mouse $(\boldsymbol{A})$ throughout the brain or $(\boldsymbol{C})$ in cortical areas only. $\boldsymbol{B}$, Decreased intersubject similarity in the Shank $3 B^{-1-}$ cohort compared with control mice. $\boldsymbol{D}$, Idiosyncratic homotopic connectivity was particularly apparent in the neocortex of Shank3B-1- mice. Error bars represent SEM. ${ }^{* *} p<0.01,{ }^{* * *} p<0.001$.
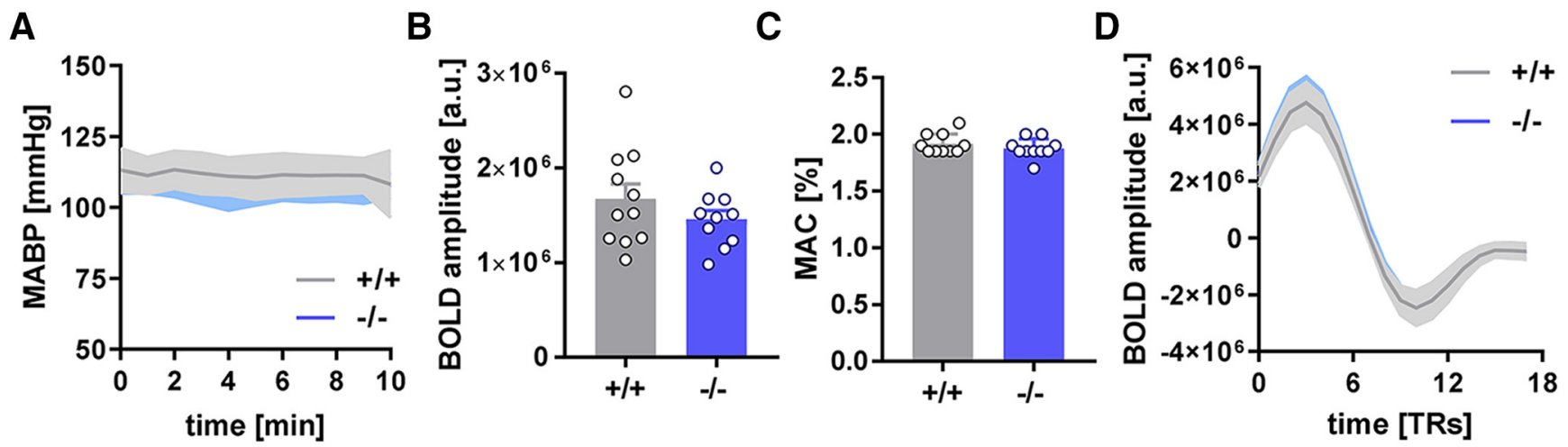

Figure 5. Sensitivity to anesthetic and hemodynamic response function. Anesthesia sensitivity as measured with $(\boldsymbol{A})$ mean arterial blood pressure mapping, $(\boldsymbol{B})$ amplitude of cortical BOLD signal fluctuations in the primary motor cortex (M1), and (C) by recording minimal alveolar concentration of halothane in Shank3B control $(+/+)$ and mutant mice $(-/-)$. D, Hemodynamic response function in the PFC of Shank3B control $(+/+)$ and mutant mice $(-/-)$. Error bars represent SEM.

autism-associated risk factors, such as agenesis of corpus callosum (Sforazzini et al., 2016), or genetic mutations involving presynaptic scaffolding genes (Michetti et al., 2017). Together, these results point at disruption of prefrontal connectivity as a prominent cross-mutational signature shared by heterogeneous ASDrelated etiologies. However, in keeping with the connectional heterogeneity observed in human investigation of ASD connectivity (Hull et al., 2017), other forms of network alterations are likely to play an equally prominent role in autism. For example, mice harboring a mutation in Chd8 gene show functional overconnectivity in hippocampal and sensory regions (Suetterlin et al., 2018), whereas Engrail-2-deficient mice (Chelini et al., 2019) and Fragile-X mouse models are characterized by reduced synchronization of somatosensory and auditory cortices (Haberl et al., 2015). Together, these initial findings suggest that different ASD-related etiologies can give rise to diverse (even diverging), yet classifiable connectivity fingerprints, substantiating a key mechanistic contribution of genetic variability to the manifold functional signatures so far described in human ASD.

The observation of reduced prefrontal connectivity in Shank $3 B^{-1-}$ mice opens the way to targeted investigations of the specific neural brain mechanisms underlying this functional impairment, and its significance for the manifestation of ASD symptoms. Given the critical contribution of fast-spiking interneurons 
A

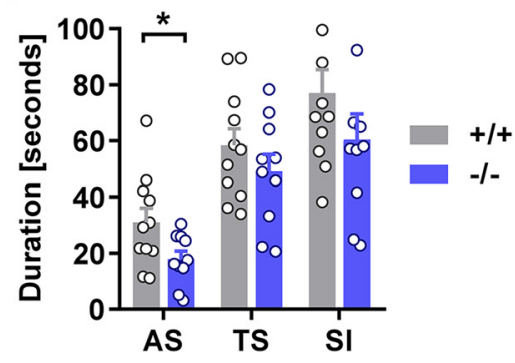

B

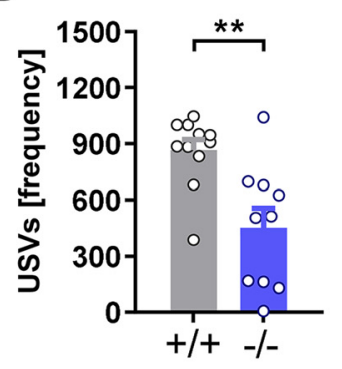

C

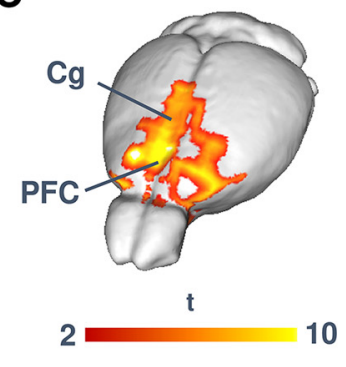

D

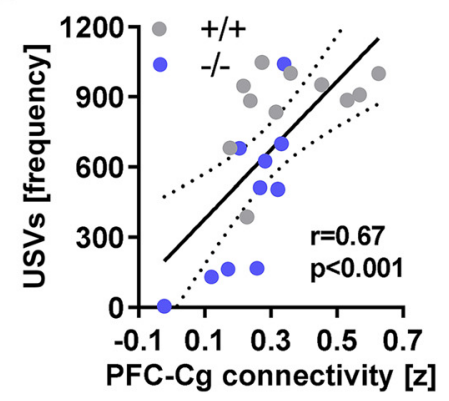

Figure 6. Correlation between prefrontal connectivity and socio-communicative dysfunction. $A$, Anogenital sniffing (AS), total sniffing (TS), and social investigation (SI) in Shank3B ${ }^{-1-}$ mutants and Shank $3 B^{+/+}$control mice. ${ }^{*} p<0.05$ (uncorrected). $\boldsymbol{B}$, Reduced USV frequency in Shank $3 B^{-1-}$ mice. C, Voxelwise correlation mapping revealed a significant positive correlation between prefrontal rsfMRI connectivity and vocalization frequency. ${ }^{* *} p<0.01$ (FDR-corrected). D, Quantification of this relationship in the PFC. Cg, Cingulate cortex. ${ }^{*} p<0.05$, ${ }^{* *} p<0.01$. Error bars represent SEM.

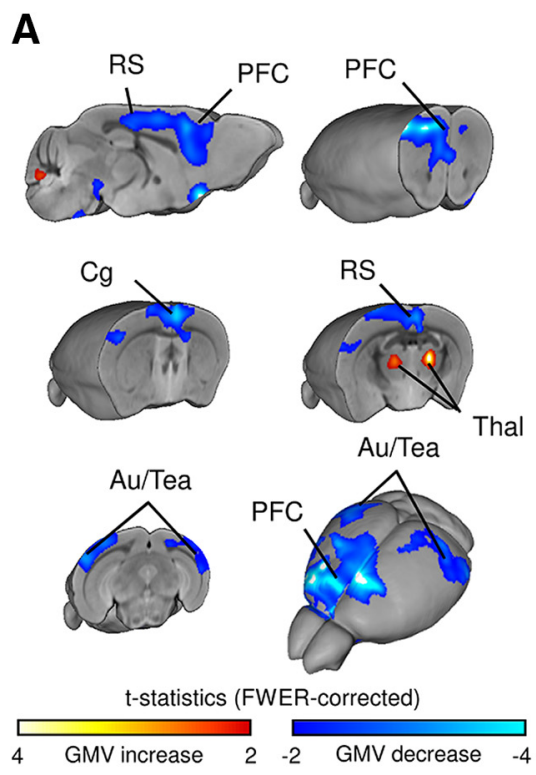

B
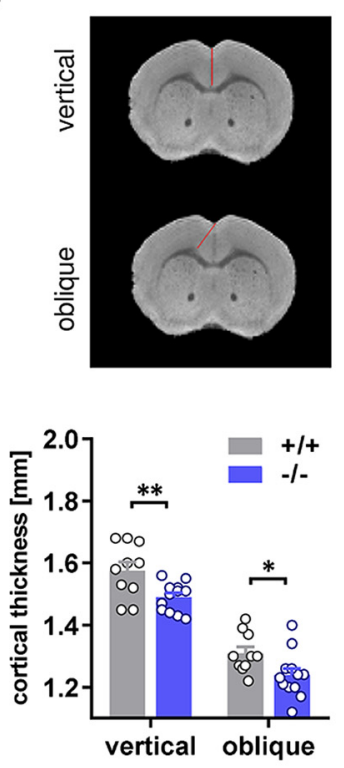
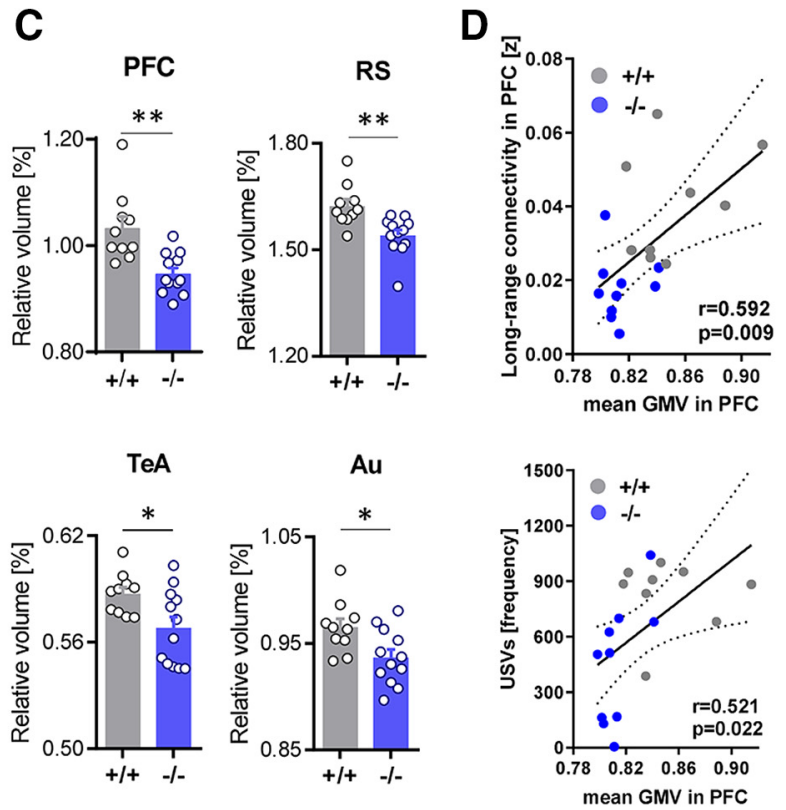

Figure 7. Reduced GM volume in prefrontal regions of Shank3B ${ }^{-1-}$ mice. $A$, Structural MRI revealed a prominent reduction of GM volume in prefrontal and retrosplenial cortices of Shank $3 B^{-1-}$ mutants. Statistically significant volume reductions were also apparent in perihippocampal associative areas. $\boldsymbol{B}$, Manual measurement of prefrontal cortical thickness on a representative high-resolution MRI slice. C, Regional volumetric analysis of GM in control and mutant Shank3B mice. Error bars represent SEM. D, Correlation between long-range rsfMRI connectivity (top) or USVs (bottom) and GM volume in PFC. Au, Auditory cortex; Cg, cingulate cortex; PFC, prefrontal cortex; RS, retrosplenial cortex; TeA, temporal association area; Thal, thalamus. * $p<0.05$, ${ }^{* *} p<0.01$.

in orchestrating long-range functional synchronization (Sohal et al., 2009), the presence of parvalbumin-positive interneuron dysfunction in Shank3B ${ }^{-/-}$mice (Gogolla et al., 2014; Filice et al., 2016) serves as a putative neurophysiological correlate for the observed fMRI desynchronization. This hypothesis is consistent with our observation of similarly impaired long-range prefrontal connectivity in Cntanp2-null mice, a mouse line exhibiting ASDlike behaviors and characterized by reduced GABA interneuron density (Liska et al., 2018). Interestingly, as previously observed in Cntnap2 mutants, our results show that Shank3B deficiency leads to a reduced number of locally projecting excitatory neurons in the PFC. While the exact mechanisms leading to improper corticofrontal circuit assembly remain to be firmly established, it has been recently shown that interneurondependent homeostatic mechanisms taking place during development can critically sculpt cortical excitatory networks (Wong et al., 2018), hence putatively implicating dysfunc- tional GABAergic maturation in the defective wiring observed in these two genetic models of ASD. Such developmental mechanisms, together with imbalances in the excitatory output of specific regional districts (Peixoto et al., 2016), could conceivably lead to permanent imbalances in interregional connectivity and synchronization.

The presence of reduced GM volume in the same prefrontal regions exhibiting dysfunctional coupling recapitulates neuroimaging observation in ASD patient cohorts, in which reduced GM volume in the temporal lobe was associated with locally decreased functional connectivity (Mueller et al., 2013). This abnormality does not appear to reflect genotype-dependent differences in neuronal density or major histological alterations in the PFC ofSHANK3-deficient mice, and is therefore more likely to reflect a reduced functional engagement of these cortical substrates as a result of their abnormal connectivity. In keeping with this notion, morphological investigations of GM volume changes detected by MRI in rodents have revealed a 
A

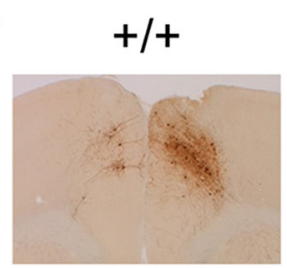

C
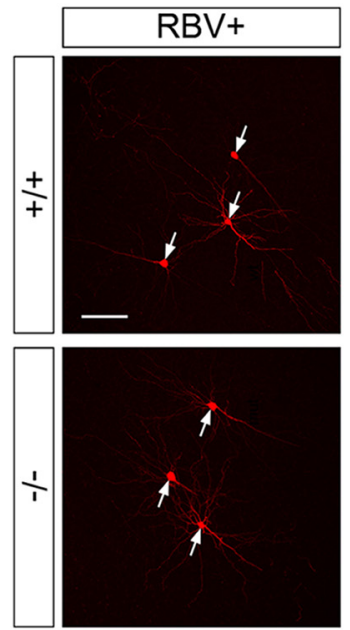

$-/-$
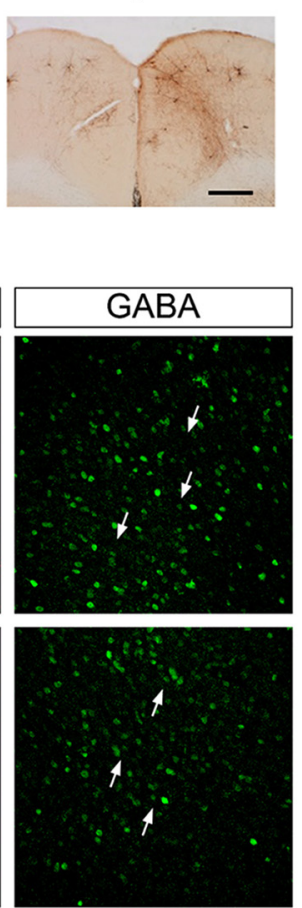

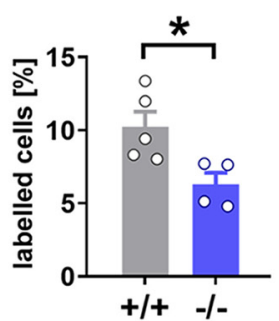

B
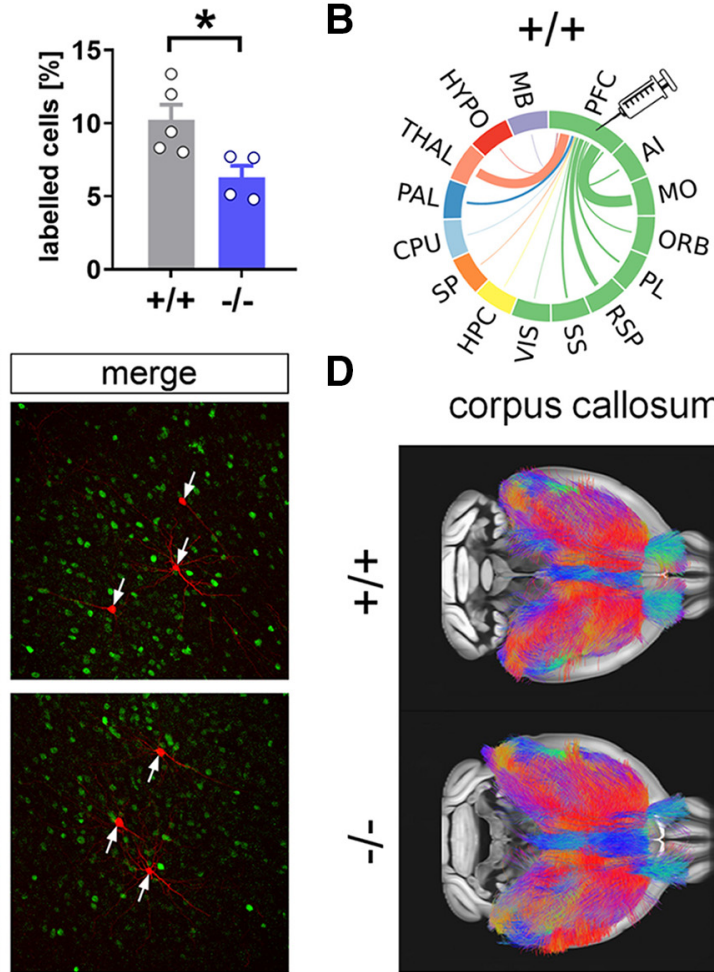

corpus callosum

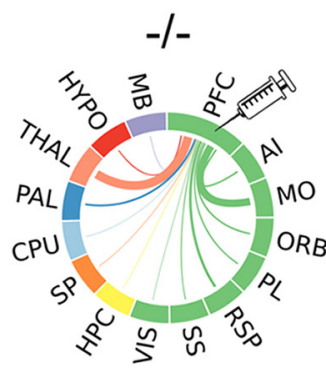

cingulate

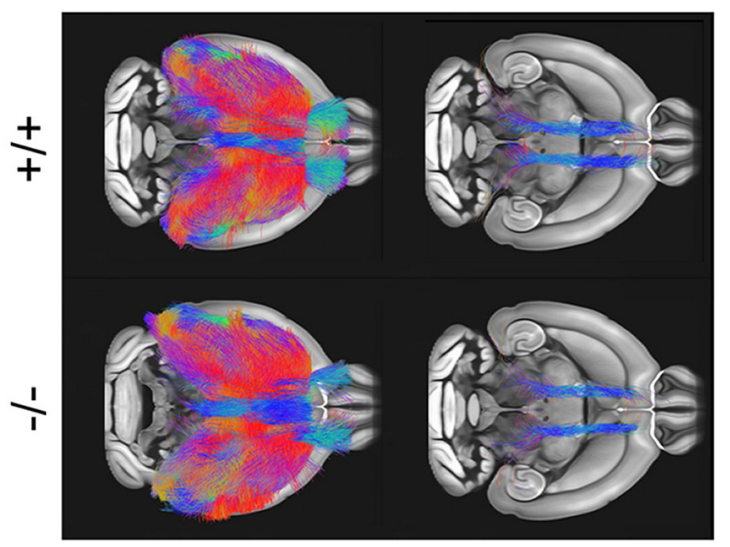

Figure 8. Neural rewiring in the PFC of Shank3B- I- mice. $A$, Distribution of retrogradely labeled cells in the PFC (bregma $1.25 \mathrm{~mm}$ ) of representative Shank3B ${ }^{-/-}$and Shank $3 B^{+/+}$control mice. Right, Regional quantification of retrogradely labeled cells in the same prefrontal areas exhibiting reduced local rsfMRI functional connectivity (Fig. 1B). ${ }^{*} p<0.05$. Error bars represent SEM. $B$, Circular layout showing the relative number of retrogradely labeled cells for each genotype. The thickness of each links is proportional to the relative number of labeled cells. Each subcortical area is depicted with a unique color. Green represents cortical areas. C, Representative confocal images showing rabies virus retrograded neurons (RBV ${ }^{+}$, left) and GABA immunostaining (GABA, middle) of Shank $3 B^{+/+}$and Shank $3 B^{-1-}$ coronal sections in the PFC. RBV ${ }^{+}$neurons are predominantly GABA-negative (merge, right). Scale bar, $100 \mu \mathrm{m}$. $D$, Preserved white matter fiber organization in Shank $3 B^{-1-}$ mice upon virtual tractographic dissection of the corpus callosum (left) and cingulum (right). Al, Agranular insular cortex; M0, motor cortex; ORB, orbital cortex; PL, prelimbic cortex; RSP, retrosplenial cortex; SS, somatosensory cortex; VIS, visual cortex; HPC, hippocampus; SP, cortical subplate; CPU, striatum; PAL, globus pallidus; THAL, thalamus; HYPO, hypothalamus; MB, midbrain.

direct link between imaging findings and dendritic spine density (Keifer et al., 2015). Based on these results, the observed reduction in GM volume could tentatively be linked to the reduced dendritic spine density previously reported in different mouse models of SHANK3 deficiency (Peca et al., 2011; Wang et al., 2016). Future investigations of the microstructure of the PFC with respect to other regional districts may be informative as to the nature of this macroscopic GM abnormality.

The presence of functional prefrontal alterations in Shank3deficient mutants extends our knowledge of the neural circuitry disrupted by mutations in this autism-risk gene, and shifts the focus from the basal ganglia, in which the role of SHANK3 deficiency has been compellingly elucidated (Peca et al., 2011; Peixoto et al., 2016), to integrative higher-order cortical regions of relevance for ASD. The role of the PFC as a crucial mediator of social recognition in rodents has prompted recent investigations of cortical dysfunction in Shank3-deficient mice, with initial results that are in excellent agreement with our findings. For example, Duffney et al. (2015) recently demonstrated that reduced social preference in Shank ${ }^{+/ \Delta C}$ mice is associated with diminished NMDA receptor function and distribution in prefrontal regions. In the same mouse model, social deficits were ameliorated by treatment with a histone deacetylase inhibitor, highlighting a contribution of epigenetic mechanisms to the expression of social deficits in SHANK3 deficiency (Qin et al., 2018). Our results are in keeping with these observations and suggest that the prefrontal dysfunction that characterizes SHANK3-deficient mice affects complex socio-communicative behavior via a large- scale involvement of distributed frontostriatal and frontocortical substrates. Notably, we found the latter connectional deficits to be tightly linked to socio-communicative function as assessed with USVs during a social interaction task. Although only correlative, this finding pinpoints a putative large-scale circuit for the expression of social communication, and strengthens translational research of SHANK3 deficiency by showing that communication deficits, one of the foremost disabilities observed in Phelan-McDermid syndrome and related shankopathies, can be recapitulated in rodent models. Interestingly, studies in rodents have shown a critical role of motor-sensory cortical areas in the expression of distress-related vocalizations, a set of regions that, however, appears to exhibit unaltered patterns of functional connectivity in our study (Sia et al., 2013). These results suggest that the communication deficits produced by SHANK3 deficiency are a top-down consequence of impaired social recognition, as opposed to primary dysfunction of motor-sensory areas related to the expression of vocalizations.

In conclusion, our work shows that SHANK3 deficiency leads to disrupted prefrontal functional connectivity, an effect associated with aberrant neuronal wiring, reduced frontocortical GM volume, and impaired social communications. Our findings suggest that Shank3 deletion may predispose to neurodevelopmental disorders and autism through dysregulation of connectivity in higher-order cortical areas, and provide a translational model for investigating connectional perturbations associated with ASD and related developmental disorders. 


\section{References}

Bertero A, Liska A, Pagani M, Parolisi R, Masferrer ME, Gritti M, Pedrazzoli M, Galbusera A, Sarica A, Cerasa A, Buffelli M, Tonini R, Buffo A, Gross C, Pasqualetti M, Gozzi A (2018) Autism-associated 16p11.2 microdeletion impairs prefrontal functional connectivity in mouse and human Brain 141:2055-2065.

Bey AL, Wang X, Yan H, Kim N, Passman RL, Yang Y, Cao X, Towers AJ, Hulbert SW, Duffney LJ, Gaidis E, Rodriguiz RM, Wetsel WC, Yin HH, Jiang YH (2018) Brain region-specific disruption of Shank3 in mice reveals a dissociation for cortical and striatal circuits in autism-related behaviors. Transl Psychiatry 8:94.

Cavaccini A, Gritti M, Giorgi A, Locarno A, Heck N, Migliarini S, Bertero A, Mereu M, Margiani G, Trusel M, Catelani T, Marotta R, De Luca MA, Caboche J, Gozzi A, Pasqualetti M, Tonini R (2018) Serotonergic signaling controls input-specific synaptic plasticity at striatal circuits. Neuron 98:801-816.e7.

Chelini G, Zerbi V, Cimino L, Grigoli A, Markicevic M, Libera F, Robbiati S, Gadler M, Bronzoni S, Miorelli S, Galbusera A, Gozzi A, Casarosa S, Provenzano G, Bozzi Y (2019) Aberrant somatosensory processing and connectivity in mice lacking Engrailed-2. J Neurosci 39:1525-1538.

Cole MW, Pathak S, Schneider W (2010) Identifying the brain's most globally connected regions. Neuroimage 49:3132-3148.

de la Torre-Ubieta L, Won H, Stein JL, Geschwind DH (2016) Advancing the understanding of autism disease mechanisms through genetics. Nat Med 22:345-361.

Di Martino A, Scheres A, Margulies DS, Kelly AM, Uddin LQ, Shehzad Z, Biswal B, Walters JR, Castellanos FX, Milham MP (2008) Functional connectivity of human striatum: a resting state fMRI study. Cereb Cortex 18:2735-2747.

Dodero L, Damiano M, Galbusera A, Bifone A, Tsaftsaris SA, Scattoni ML, Gozzi A (2013) Neuroimaging evidence of major morpho-anatomical and functional abnormalities in the BTBR T $+\mathrm{TF} / \mathrm{J}$ mouse model of autism. PLoS One 8:e76655.

Dong HW (2008) The Allen reference atlas: a digital color brain atlas of the C57BL/6J male mouse. New York: Wiley.

Duffney LJ, Zhong P, Wei J, Matas E, Cheng J, Qin L, Ma K, Dietz DM, Kajiwara Y, Buxbaum JD, Yan Z (2015) Autism-like deficits in Shank3deficient mice are rescued by targeting actin regulators. Cell Rep 11:14001413.

Ferrari L, Turrini G, Crestan V, Bertani S, Cristofori P, Bifone A, Gozzi A (2012) A robust experimental protocol for pharmacological fMRI in rats and mice. J Neurosci Methods 204:9-18.

Filice F, Vörckel KJ, Sungur AÖ, Wöhr M, Schwaller B (2016) Reduction in parvalbumin expression not loss of the parvalbumin-expressing GABA interneuron subpopulation in genetic parvalbumin and shank mouse models of autism. Mol Brain 9:10.

Gogolla N, Takesian AE, Feng G, Fagiolini M, Hensch TK (2014) Sensory integration in mouse insular cortex reflects GABA circuit maturation. Neuron 83:894-905.

Gozzi A, Schwarz AJ (2016) Large-scale functional connectivity networks in the rodent brain. Neuroimage 127:496-509.

Haberl MG, Zerbi V, Veltien A, Ginger M, Heerschap A, Frick A (2015) Structural-functional connectivity deficits of neocortical circuits in the Fmr1 (-/y) mouse model of autism. Sci Adv 1:e1500775.

Hahamy A, Behrmann M, Malach R (2015) The idiosyncratic brain: distortion of spontaneous connectivity patterns in autism spectrum disorder. Nat Neurosci 18:302-309.

Hull JV, Dokovna LB, Jacokes ZJ, Torgerson CM, Irimia A, Van Horn JD (2017). Resting-state functional connectivity in autism spectrum disorders: A review. Frontiers in psychiatry 7:205.

Janke AL, Ullmann JF, Kurniawan ND, Paxinos G, Keller M, Yang Z, Richards K, Egan G, Petrou S, Galloway G (2012) $15 \mu$ m average mouse models in Waxholm space from 16.4 T $30 \mu \mathrm{m}$ images, p 4191. In: 20th Annual ISMRM Scientific Meeting and Exhibition, Melbourne, Australia.

Jenkinson M, Beckmann CF, Behrens TE, Woolrich MW, Smith SM (2012) FSL. Neuroimage 62:782-790.

Keifer OP Jr, Hurt RC, Gutman DA, Keilholz SD, Gourley SL, Ressler KJ (2015) Voxel-based morphometry predicts shifts in dendritic spine density and morphology with auditory fear conditioning. Nat Commun 6:7582.

Leblond CS, Nava C, Polge A, Gauthier J, Huguet G, Lumbroso S, Giuliano F, Stordeur C, Depienne C, Mouzat K, Pinto D, Howe J, Lemière N, Durand
CM, Guibert J, Ey E, Toro R, Peyre H, Mathieu A, Amsellem F, et al. (2014) Meta-analysis of SHANK mutations in autism spectrum disorders: a gradient of severity in cognitive impairments. PLoS Genet 10:e1004580.

Liska A, Gozzi A (2016) Can mouse imaging studies bring order to autism connectivity chaos? Front Neurosci 10:484.

Liska A, Galbusera A, Schwarz AJ, Gozzi A (2015) Functional connectivity hubs of the mouse brain. Neuroimage 115:281-291.

Liska A, Bertero A, Gomolka R, Sabbioni M, Galbusera A, Barsotti N, Panzeri S, Scattoni ML, Pasqualetti M, Gozzi A (2018) Homozygous loss of autism-risk gene CNTNAP2 results in reduced local and long-range prefrontal functional connectivity. Cereb Cortex 10:1-13.

Liu X, Zhu XH, Zhang Y, Chen W (2011) Neural origin of spontaneous hemodynamic fluctuations in rats under burst-suppression anesthesia condition. Cereb Cortex 21:374-384.

Mei Y, Monteiro P, Zhou Y, Kim JA, Gao X, Fu Z, Feng G (2016) Adult restoration of Shank3 expression rescues selective autistic-like phenotypes. Nature 530:481-484.

Michetti C, Caruso A, Pagani M, Sabbioni M, Medrihan L, David G, Galbusera A, Morini M, Gozzi A, Benfenati F, Scattoni ML (2017) The knockout of synapsin II in mice impairs social behavior and functional connectivity generating an ASD-like phenotype. Cereb Cortex 27:50145023 .

Monteiro P, Feng G (2017) SHANK proteins: roles at the synapse and in autism spectrum disorder. Nat Rev Neurosci 18:147-157.

Mueller S, Keeser D, Samson AC, Kirsch V, Blautzik J, Grothe M, Erat O, Hegenloh M, Coates U, Reiser MF, Hennig-Fast K, Meindl T (2013) Convergent findings of altered functional and structural brain connectivity in individuals with high functioning autism: a multimodal MRI study. PLoS One 8:e67329.

Pagani M, Bifone A, Gozzi A (2016a) Structural covariance networks in the mouse brain. Neuroimage 129:55-63.

Pagani M, Damiano M, Galbusera A, Tsaftaris SA, Gozzi A (2016b) Semiautomated registration-based anatomical labelling, voxel based morphometry and cortical thickness mapping of the mouse brain. J Neurosci Methods 267:62-73.

Peca J, Feliciano C, Ting JT, Wang W, Wells MF, Venkatraman TN, Lascola CD, Fu Z, Feng G (2011) Shank3 mutant mice display autistic-like behaviours and striatal dysfunction. Nature 472:437-442.

Peixoto RT, Wang W, Croney DM, Kozorovitskiy Y, Sabatini BL (2016) Early hyperactivity and precocious maturation of corticostriatal circuits in Shank3B ${ }^{-1-}$ mice. Nat Neurosci 19:716-724.

Phelan K, McDermid HE (2012) The 22q13. 3 deletion syndrome (PhelanMcDermid syndrome). Mol Syndromol 2:186-201.

Pucilowska J, Vithayathil J, Pagani M, Kelly C, Karlo JC, Robol C, Morella I, Gozzi A, Brambilla R, Landreth GE (2018) Pharmacological inhibition of ERK signaling rescues pathophysiology and behavioral phenotype associated with 16p11.2 chromosomal deletion in mice. J Neurosci 38: $6640-6652$

Qin L, Ma K, Wang ZJ, Hu Z, Matas E, Wei J, Yan Z (2018) Social deficits in Shank3-deficient mouse models of autism are rescued by histone deacetylase (HDAC) inhibition. Nat Neurosci 21:564-575.

Rudie JD, Hernandez LM, Brown JA, Beck-Pancer D, Colich NL, Gorrindo P, Thompson PM, Geschwind DH, Bookheimer SY, Levitt P, Dapretto M (2012) Autism-associated promoter variant in MET impacts functional and structural brain networks. Neuron 75:904-915.

Sannino T, Gozzi A, Cerasa A, Schegga D, Manago F, Damiano M, Galbusera A, Dodero L, Tonelli D, Bifone A, Tsaftaris AS, Weinberger DR, Spalletta G, Papaleo F (2013) Sexual dimorphisms in COMT modulation of cortical anatomy and behavior in mice and humans. Cereb Cortex 25:25292541.

Scattoni ML, Gandhy SU, Ricceri L, Crawley JN (2008) Unusual repertoire of vocalizations in the BTBR $\mathrm{T}+\mathrm{tf} / \mathrm{J}$ mouse model of autism. PLoS One 3:e3067.

Scattoni ML, Ricceri L, Crawley JN (2011) Unusual repertoire of vocalizations in adult BTBR $\mathrm{T}+\mathrm{tf} / \mathrm{J}$ mice during three types of social encounters. Genes Brain Behav 10:44-56.

Scattoni ML, Martire A, Cartocci G, Ferrante A, Ricceri L (2013) Reduced social interaction, behavioural flexibility and BDNF signalling in the BTBR T+tf/J strain, a mouse model of autism. Behav Brain Res 251:3540.

Schindelin J, Arganda-Carreras I, Frise E, Kaynig V, Longair M, Pietzsch T, 
Preibisch S, Rueden C, Saalfeld S, Schmid B, Tinevez JY, White DJ, Hartenstein V, Eliceiri K, Tomancak P, Cardona A (2012) Fiji: an opensource platform for biological-image analysis. Nat Methods 9:676-682.

Scott-Van Zeeland AA, Abrahams BS, Alvarez-Retuerto AI, Sonnenblick LI, Rudie JD, Ghahremani D, Mumford JA, Poldrack RA, Dapretto M, Geschwind DH, Bookheimer SY (2010) Altered functional connectivity in frontal lobe circuits is associated with variation in the autism risk gene CNTNAP2. Sci Transl Med 2:56ra80.

Sforazzini F, Schwarz AJ, Galbusera A, Bifone A, Gozzi A (2014) Distributed $\mathrm{BOLD}$ and $\mathrm{CBV}$-weighted resting-state networks in the mouse brain. Neuroimage 87:403-415.

Sforazzini F, Bertero A, Dodero L, David G, Galbusera A, Scattoni ML, Pasqualetti M, Gozzi A (2016) Altered functional connectivity networks in acallosal and socially impaired BTBR mice. Brain Struct Funct 221:941-954.

Sia GM, Clem RL, Huganir RL (2013) The human language-associated gene SRPX2 regulates synapse formation and vocalization in mice. Science 342:987-991.

Smith SM, Jenkinson M, Johansen-Berg H, Rueckert D, Nichols TE, Mackay CE, Watkins KE, Ciccarelli O, Cader MZ, Matthews PM, Behrens TE (2006) Tract-based spatial statistics: voxelwise analysis of multi-subject diffusion data. Neuroimage 31:1487-1505.

Sohal VS, Zhang F, Yizhar O, Deisseroth K (2009) Parvalbumin neurons and gamma rhythms enhance cortical circuit performance. Nature 459:698-702.

Steffey MA, Brosnan RJ, Steffey EP (2003) Assessment of halothane and sevoflurane anesthesia in spontaneously breathing rats. Am J Vet Res 64:470-474.

Suetterlin P, Hurley S, Mohan C, Riegman KL, Pagani M, Caruso A, Ellegood J, Galbusera A, Crespo-Enriquez I, Michetti C, Yee Y, Ellingford R, Brock O, Delogu A, Francis-West P, Lerch JP, Scattoni ML, Gozzi A, Fernandes C, Basson MA (2018) Altered neocortical gene expression, brain overgrowth and functional over-connectivity in Chd8 haploinsufficient mice. Cereb Cortex 28:2192-2206.

Tabet AC, Rolland T, Ducloy M, Lévy J, Buratti J, Mathieu A, Haye D, Perrin L, Dupont C, Passemard S, Capri Y, Verloes A, Drunat S, Keren B, Mignot C, Marey I, Jacquette A, Whalen S, Pipiras E, Benzacken B, et al. (2017) A framework to identify contributing genes in patients with PhelanMcDermid syndrome. NPJ Genom Med 2:32.

Tournier JD, Calamante F, Connelly A (2012) MRtrix: diffusion tractography in crossing fiber regions. Int J Imag Syst Technol 22:53-66.

Van Den Heuvel MP, Hulshoff Pol HE (2010) Exploring the brain network: a review on resting-state fMRI functional connectivity. Eur Neuropsychopharmacol 20:519-534.

Vasa RA, Mostofsky SH, Ewen JB (2016) The disrupted connectivity hypothesis of autism spectrum disorders: time for the next phase in research. Biol Psychiatry Cogn Neurosci Neuroimaging 1:245-252.

Vicidomini C, Ponzoni L, Lim D, Schmeisser MJ, Reim D, Morello N, Orellana D, Tozzi A, Durante V, Scalmani P, Mantegazza M, Genazzani AA, Giustetto M, Sala M, Calabresi P, Boeckers TM, Sala C, Verpelli C (2017) Pharmacological enhancement of mGlu5 receptors rescues behavioral deficits in SHANK3 knock-out mice. Mol Psychiatry 22:689-702.

Wang X, Bey AL, Katz BM, Badea A, Kim N, David LK, Duffney LJ, Kumar S, Mague SD, Hulbert SW, Dutta N, Hayrapetyan V, Yu C, Gaidis E, Zhao S, Ding JD, Xu Q, Chung L, Rodriguiz RM, Wang F, et al. (2016) Altered mGluR5-homer scaffolds and corticostriatal connectivity in a Shank3 complete knockout model of autism. Nat Commun 7:11459.

Winkler AM, Ridgway GR, Webster MA, Smith SM, Nichols TE (2014) Permutation inference for the general linear model. Neuroimage 92:381-397.

Wong FK, Bercsenyi K, Sreenivasan V, Portalés A, Fernández-Otero M, Marín O (2018) Pyramidal cell regulation of interneuron survival sculpts cortical networks. Nature 557:668-673.

Worsley KJ, Evans AC, Marrett S, Neelin P (1992) A three-dimensional statistical analysis for CBF activation studies in human brain. J Cereb Blood Flow Metab 12:900-918.

Wu GR, Liao W, Stramaglia S, Ding JR, Chen H, Marinazzo D (2013) A blind deconvolution approach to recover effective connectivity brain networks from resting state fMRI data. Med Image Anal 17:365-374.

Yushkevich PA, Piven J, Hazlett HC, Smith RG, Ho S, Gee JC, Gerig G (2006) User-guided 3D active contour segmentation of anatomical structures: significantly improved efficiency and reliability. Neuroimage 31:11161128 .

Zalesky A, Fornito A, Bullmore ET (2010) Network-based statistic: identifying differences in brain networks. Neuroimage 53:1197-1207.

Zhan Y, Paolicelli RC, Sforazzini F, Weinhard L, Bolasco G, Pagani F, Vyssotski AL, Bifone A, Gozzi A, Ragozzino D, Gross CT (2014) Deficient neuron-microglia signaling results in impaired functional brain connectivity and social behavior. Nat Neurosci 17:400-406.

Zhao H, Tu Z, Xu H, Yan S, Yan H, Zheng Y, Yang W, Zheng J, Li Z, Tian R, Lu Y, Guo X, Jiang YH, Li XJ, Zhang YQ (2017) Altered neurogenesis and disrupted expression of synaptic proteins in prefrontal cortex of SHANK3-deficient nonhuman primate. Cell Res 27:1293-1297. 